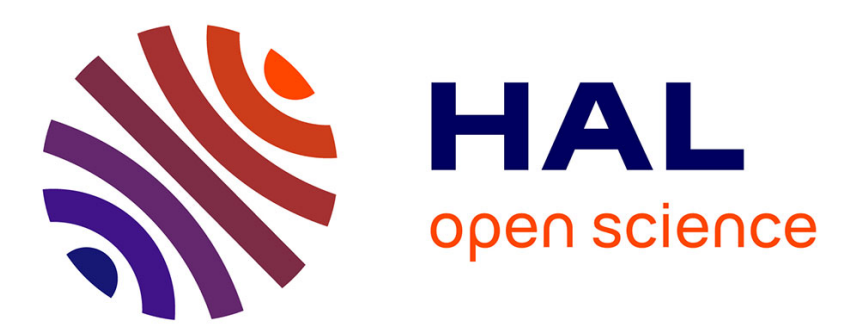

\title{
Minimal time crisis versus minimum time to reach a viability kernel: a case study in the prey-predator model
} Térence Bayen, Alain Rapaport

\section{To cite this version:}

Térence Bayen, Alain Rapaport. Minimal time crisis versus minimum time to reach a viability kernel: a case study in the prey-predator model. Optimal Control Applications and Methods, 2019, 40 (2), pp.330-350. 10.1002/oca.2484 . hal-01943636v1

\section{HAL Id: hal-01943636 \\ https://hal.science/hal-01943636v1}

Submitted on 4 Dec 2018 (v1), last revised 4 Oct 2020 (v2)

HAL is a multi-disciplinary open access archive for the deposit and dissemination of scientific research documents, whether they are published or not. The documents may come from teaching and research institutions in France or abroad, or from public or private research centers.
L'archive ouverte pluridisciplinaire HAL, est destinée au dépôt et à la diffusion de documents scientifiques de niveau recherche, publiés ou non, émanant des établissements d'enseignement et de recherche français ou étrangers, des laboratoires publics ou privés. 


\title{
Minimal time crisis versus minimal time to reach a viability kernel
}

\author{
Terence Bayen*, Alain Rapaport ${ }^{\dagger}$
}

April 11, 2018

\begin{abstract}
In this work, we provide a reformulation of the minimal time crisis problem associated to a given domain as a free terminal time control problem. This is made possible supposing that the viability kernel is nonempty, and that it is reachable from the state space. Moreover, an additional hypothesis on consecutive crossing time of the constraint set is required. Thanks to this result, we compute an optimal synthesis for the minimal time crisis problem governed by the Lotka-Volterra prey-predator model, with a controlled mortality on the predators. Finally, we compare the time spent in the crisis set by optimal trajectories of the minimal time crisis problem and the minimal time problem to reach the viability kernel.
\end{abstract}

Keywords. Optimal Control, Viability Theory, Pontryagin Maximum Principle, Lotka-Volterra system.

\section{Introduction}

\section{Optimality conditions for the time crisis function}

In this section, we provide optimality conditions on the time crisis function in a general setup. We first introduce some notations. Given a non-empty subset $C$ of $\mathbb{R}^{n}$, we denote by $\operatorname{Int}(C)$ its interior and by $\partial C$ its boundary. We also denote by $|\cdot|$ the euclidean norm in $\mathbb{R}^{n}, n \geq 1$. If $C \subset \mathbb{R}^{n}, n \geq 1$ is a non-empty closed convex subset of $\mathbb{R}^{n}$, the normal cone to $C$ at a point $x \in C$ is defined as

$$
N_{C}(x):=\left\{p \in \mathbb{R}^{n} ; p \cdot(y-x) \leq 0, \forall y \in C\right\},
$$

where $a \cdot b$ denotes the standard scalar product of two vectors $a, b \in \mathbb{R}^{n}$. Throughout this section, we consider a control system:

$$
\dot{x}=f(x, u),
$$

where $f: \mathbb{R}^{n} \times \mathbb{R}^{m} \rightarrow \mathbb{R}^{n}$ is the dynamics, $x$ is the state, and $u$ the control that takes values in a non-empty closed subset $U$ of $\mathbb{R}^{m}$. The admissible control set is classically

$$
\mathcal{U}:=\{u:[0,+\infty) \rightarrow U ; u \text { meas. }\}
$$

We assume the usual regularity assumptions on the dynamics (see e.g. [12]):

(H1) The function $f$ is continuous w.r.t. $(x, u)$, of class $C^{1}$ w.r.t. $x$ and satisfies the linear growth condition: there exist $c_{1}>0$ and $c_{2}>0$ such that for all $x \in \mathbb{R}^{n}$ and all $u \in U$, one has:

$$
|f(x, u)| \leq c_{1}|x|+c_{2}
$$

(H2) For any $x \in \mathbb{R}^{n}$, the velocity set $F(x):=\{f(x, u) ; u \in U\}$ is a non-empty compact convex set.

${ }^{*}$ IMAG, Univ Montpellier, CNRS, Montpellier, France and MISTEA, Univ Montpellier, INRA, Montpellier SupAgro, France. terence.bayen@umontpellier.fr

${ }^{\dagger}$ MISTEA, Univ Montpellier, INRA, Montpellier SupAgro, France. alain.rapaport@inra.fr 
Under hypotheses (H1)-(H2), for any initial condition $x_{0} \in \mathbb{R}^{n}$ and any $T \geq 0$, there exists a unique absolutely continuous solution of $(2.1)$ defined over $[0, T]$ such that $x(0)=x_{0}$, denoted by $x_{u}\left(\cdot, x_{0}\right)$ hereafter (this is a consequence of Cauchy-Lipschitz's Theorem). Given a closed subset $K$ of $\mathbb{R}^{n}$ (that plays the role of constraint set), let us now introduce the viability kernel of $K$ under the dynamics $f$ as

$$
\operatorname{Viab}(K):=\left\{x_{0} \in K ; \exists u \in \mathcal{U}, x_{u}\left(t, x_{0}\right) \in K, \forall t \geq 0\right\}
$$

If non-empty, the viability kernel of $K$ is a closed subset of $K$ and it enjoys several properties. In particular, it can be reached from outside only at its boundary in common with the boundary of the set $K$. We refer to [2, 3] for more details on this set and its fundamental role in several economic and biological models. Hereafter, we are interested in studying properties of the minimal time crisis function $T: \mathbb{R}^{n} \rightarrow \mathbb{R}_{+} \cup\{+\infty\}$ which states as

$$
T\left(x_{0}\right):=\inf _{u \in \mathcal{U}} \int_{0}^{+\infty} \mathbb{1}_{K^{c}}\left(x_{u}\left(t, x_{0}\right)\right) \mathrm{d} t,
$$

where $\mathbb{1}_{K^{c}}$ is the indicator function of $K^{c}$ :

$$
\mathbb{1}_{K^{c}}(x):=\left\{\begin{array}{lll}
0 & \text { if } & x \in K \\
1 & \text { if } \quad x \notin K
\end{array}\right.
$$

and $K^{c}:=\mathbb{R}^{n} \backslash K$ denotes the complementary of the set $K$. It has been introduced in [16] in the context of viability theory $[2,3]$ typically when the initial state does not belong to the viability kernel. In particular, one can show that it is a lower semi-continuous function (see $[16,4,5]$ ). Our main concern in this section is to investigate a reformulation of (2.3) over a finite horizon that involves the viability kernel. This will allow us to obtain necessary optimality conditions on the time crisis function, thanks to the hybrid maximum principle [11]. To do so, let us introduce an additional hypothesis:

(H3) The viability kernel of $K$ under the dynamics $f, V i a b(K)$ is non-empty and for any initial condition in $\mathbb{R}^{n}$, there exists a control $u \in \mathcal{U}$ steering $x_{0}$ to $\operatorname{Viab}(K)$ in finite time.

Consider now the auxiliary optimal control problem:

$$
\hat{T}\left(x_{0}\right):=\inf _{\tau \geq 0, u \in \mathcal{U}} \int_{0}^{\tau} \mathbb{1}_{K^{c}}\left(x_{u}\left(t, x_{0}\right)\right) \mathrm{d} t \quad \text { s.t. } x_{u}\left(\tau, x_{0}\right) \in \operatorname{Viab}(K) .
$$

Under the additional hypothesis (H3), the existence of an optimal control for both problems (2.3) and (2.4) follows from standard argumentation (see $[16,4]$ ). Moreover, for any $x_{0} \in \mathbb{R}^{n}, T\left(x_{0}\right)<+\infty$ and $\hat{T}\left(x_{0}\right)<+\infty$. In order to relate (2.3) and (2.4), we introduce the notion of crossing time.

Definition 2.1. Given a solution $x_{u}\left(\cdot, x_{0}\right)$ of $(2.1)$, we say that a time $t_{c}>0$ is a crossing time for $x_{u}\left(\cdot, x_{0}\right)$ from $K$ to $K^{c}$ if the control $u$ is left-and right-continuous at $t_{c}, x_{u}\left(t_{c}, x_{0}\right) \in \partial K$, and there exists $\eta>0$ such that for any time $t \in\left[t_{c}-\eta, t_{c}\right]$, resp. $t \in\left(t_{c}, t_{c}+\eta\right], x_{u}\left(t, x_{0}\right) \in K$, resp. $x_{u}\left(t, x_{0}\right) \in K^{c}$. Similarly, we define the notion of crossing time from $K^{c}$ to $K$.

Next, we consider the following hypothesis:

(H4) There exists a number $\eta>0$ such that for any pair of consecutive crossing times $t_{1}<t_{2}$ from $K$ to $K^{c}$ or from $K^{c}$ to $K$, one has $t_{2}-t_{1} \geq \eta$,

which allows us to state the following equivalence result.

Proposition 2.1. Suppose that (H1)-(H2)-(H3)-(H4) are satisfied. Then, for any $x_{0} \in \mathbb{R}^{n}$ one has

$$
T\left(x_{0}\right)=\hat{T}\left(x_{0}\right) .
$$

Furthermore, for any $x_{0}$, the infimum in (2.4) is reached for a finite $\tau$.

Proof. As the value functions $T$ and $\hat{T}$ are clearly identically equal to zero in $V i a b(\underline{x})$, we consider $x_{0} \notin$ $\operatorname{Viab}(K)$. It is known (see [16]) that one has $T\left(x_{0}\right) \leq \tilde{V}\left(x_{0}\right)$, where

$$
\tilde{V}\left(x_{0}\right):=\inf _{u \in \mathcal{U}} T_{u} \quad \text { s.t. } x_{u}\left(T_{u}, x_{0}\right) \in \operatorname{Viab}(K) .
$$


Moreover, Hypothesis (H3) implies $\tilde{V}\left(x_{0}\right)<+\infty$. Let $u^{*}(\cdot)$ be an optimal control for $T\left(x_{0}\right)$ and $x^{*}$ the associated solution starting from $x_{0}$. Define the time $\tau\left(x_{0}\right) \in \mathbb{R}_{+} \cup\{+\infty\}$ by:

$$
\tau\left(x_{0}\right):=\sup \left\{t \geq 0 ; x^{*}(t) \in K^{c}\right\}
$$

and suppose by contradiction that $\tau\left(x_{0}\right)=+\infty$. As $T\left(x_{0}\right)<+\infty$, there exists $t_{0} \geq 0$ such that $x^{*}\left(t_{0}\right) \in K$. Now, as $\tau\left(x_{0}\right)=+\infty$, there exists $t_{1} \geq t_{0}$ such that $t_{1}$ is a crossing time from $K$ to $K^{c}$. We now define $t_{2}$ as the first entry time $t>t_{1}$ of $x^{*}$ from $K^{c}$ into $K\left(t_{2}\right.$ exists as $\left.T\left(x_{0}\right)<+\infty\right)$. From (H4), we deduce that $t_{2}-t_{1} \geq \eta>0$. By the Dynamic Programming Principle, $x^{*}$ is also optimal from $x^{*}\left(t_{2}\right)$ and one has $T\left(x^{*}\left(t_{2}\right)\right) \leq T\left(y_{0}\right)<+\infty$ together with $\tau\left(x^{*}\left(t_{2}\right)\right)=\tau\left(y_{0}\right)=+\infty$. Therefore, the same argument can be applied from $\left(t_{2}, x^{*}\left(t_{2}\right)\right)$ and we obtain two increasing sequences of times $\left(t_{i, n}\right)_{n}, i=1$, 2 , such that one has $t_{2, n}-t_{1, n} \geq \eta>0$ for any $n \in \mathbb{N}$. It follows that one has $T\left(y_{0}\right)=+\infty$ and thus a contradiction. Therefore, we necessary have $\tau\left(x_{0}\right)<+\infty$ which implies that $x^{*}(t) \in K$ for any time $t \geq \tau\left(x_{0}\right)$ i.e. $x^{*}\left(\tau\left(x_{0}\right)\right) \in V i a b(K)$. It follows that

$$
T\left(x_{0}\right)=\int_{0}^{\tau\left(x_{0}\right)} \mathbb{1}_{K^{c}}\left(x^{*}(t)\right) \mathrm{d} t \geq \hat{T}\left(x_{0}\right),
$$

using that $x^{*}\left(\tau\left(x_{0}\right)\right) \in \operatorname{Viab}(K)$. On the other hand, let $(\hat{\tau}, \hat{u}(\cdot)) \in \mathbb{R}_{+} \times \mathcal{U}$ be an optimal pair for $\hat{T}\left(x_{0}\right)$. If $\hat{x}(\cdot)$ denotes the associated trajectory, we then have $\hat{x}(\hat{\tau}) \in \operatorname{Viab}(K)$ with $\hat{u}$ defined over $[0, \hat{\tau}]$. Hence, we can extend $\hat{u}$ to a control function $\bar{u} \in \mathcal{U}$ defined on $[0+\infty)$ such that the associated trajectory $\bar{u}(\cdot)$ satisfies $\bar{x}(t) \in \operatorname{Viab}(K)$ for any time $t \geq \hat{\tau}$. We then have

$$
\hat{T}\left(x_{0}\right)=\int_{0}^{\hat{\tau}} \mathbb{1}_{K^{c}}\left(\hat{x}(t) \mathrm{d} t=\int_{0}^{+\infty} \mathbb{1}_{K^{c}}(\bar{x}(t)) \mathrm{d} t \geq T\left(x_{0}\right),\right.
$$

and the conclusion follows.

Finally, to prove that the infimum in (2.4) is reached for a finite $\tau$, we suppose by contradiction that this is not the case. As previously, we can then find two increasing sequences of times $\left(t_{i, n}\right)_{n}, i=1,2$, such that one has $t_{2, n}-t_{1, n} \geq \eta$ for any $n \in \mathbb{N}$. Similarly, we find a contradiction with the fact that $\hat{T}\left(x_{0}\right)$ is finite, which ends the proof.

Remark 2.1. Hypothesis (H4) is about trajectories of the system. It appears to be a strong hypothesis, nevertheless, we shall see that it is satisfied in Lotka-Volterra type models, in Section 5.

In connection with Hypothesis (H4), it is relevant to recall the chattering phenomenon ${ }^{1}$, following for instance $[33,35,36]$.

Definition 2.2. Given $x_{0} \in \mathbb{R}^{n}$ and a solution $x_{u}\left(\cdot, x_{0}\right)$ of $(2.1)$ defined over $[0,+\infty)$, we say that a chattering phenomenon occurs if there exist two sequences of times $\left(t_{n}^{\text {out }}\right)_{n \geq 0},\left(t_{n}^{i n}\right)_{n \geq 0}$ satisfying:

- For any $n \in \mathbb{N}, t_{n}^{\text {out }}$ and $t_{n}^{\text {in }}$ are two consecutive crossing time for $x_{u}\left(\cdot, x_{0}\right)$ from $K$ to $K^{c}$ and from $K^{c}$ to $K$ respectively.

- For any $n \in \mathbb{N}$, one has $t_{n}^{\text {out }}-t_{n}^{\text {in }}>0$, and $t_{n}^{\text {out }}-t_{n}^{\text {in }} \rightarrow 0$ when $n \rightarrow+\infty$.

Let us finally recall the definition of transverse crossing times [4] (for convex sets $K$ ).

Definition 2.3. Given an admissible trajectory $x_{u}\left(\cdot, x_{0}\right)$, a crossing time $t_{c}$ (from $K$ to $K^{c}$ or from $K^{c}$ to $K)$ is transverse when there exists $\nu, \nu^{\prime} \in N_{K}\left(x_{u}\left(t_{c}\right)\right)$ such that $\dot{x}_{u}\left(t_{c}^{-}\right) \cdot \nu \neq 0$ and $\dot{x}_{u}\left(t_{c}^{+}\right) \cdot \nu^{\prime} \neq 0$.

In other words, a transverse crossing time $t_{c}$ is such that the trajectory does not hit the boundary of $K$ tangentially while crossing $K$ (however, the control could switch at time $t_{c}$ ). We are now in position to state optimality conditions for problem (2.3). Let $H: \mathbb{R}^{n} \times \mathbb{R}^{n} \times \mathbb{R} \times \mathbb{R}^{m} \rightarrow \mathbb{R}$ be the Hamiltonian associated to the system defined by:

$$
H=H\left(x, p, p_{0}, u\right):=p \cdot f(x, u)+p_{0} \mathbb{1}_{K^{c}}(x) .
$$

We shall now apply the hybrid principle on the minimal time crisis problem (see $[4,5,22])$.

\footnotetext{
${ }^{1}$ In general, this terminology is used when an optimal control has an infinite number of switching points in a finite horizon (in presence of a singular arc of order 2), see Fuller's example [35].
} 
Theorem 2.1. Suppose that assumptions (H1)-(H2)-(H3)-(H4) are satisfied. Let $u$ be an optimal control for (2.3) defined over an interval $\left[0, \tau_{f}\right], x$ the associated trajectory, and $n$ the number of crossing times of $x$. Set $t_{0}=0<t_{1}<\cdots<t_{n}<t_{n+1}=T$ the $n$ crossing times of $x$. We suppose in addition that each crossing time is transverse. Then, there exists a function $p:\left[0, \tau_{f}\right] \rightarrow \mathbb{R}^{n}$ which is absolutely continuous in each interval $\left(t_{i}, t_{i+1}\right), 0 \leq i \leq n-1$ and $p_{0} \leq 0$ satisfying the following conditions.

- The pair $\left(p(\cdot), p_{0}\right)$ is non-zero.

- The function $p$ satisfies the adjoint equation

$$
\dot{p}_{i}(t)=-\nabla_{x} H\left(x(t), p(t), p_{0}, u(t)\right) \quad \text { a.e. } t \in\left(t_{i}, t_{i+1}\right), \quad 0 \leq i \leq n-1 .
$$

- The control u satisfies the Hamiltonian condition

$$
u(t) \in \arg \max _{\omega \in U} H\left(x(t), p(t), p_{0}, \omega\right) \quad \text { a.e. } t \in\left[0, \tau_{f}\right] .
$$

- At every crossing time $t_{i}, 1 \leq i \leq n$, the covector satisfies:

$$
p\left(t_{i}^{+}\right)=p\left(t_{i}^{-}\right)+\frac{p\left(t_{i}^{-}\right) \cdot\left(f\left(x\left(t_{i}\right), u\left(t_{i}^{-}\right)\right)-f\left(x\left(t_{i}\right), u\left(t_{i}^{+}\right)\right)\right)+\sigma p_{0}}{n\left(x\left(t_{i}\right)\right) \cdot f\left(x\left(t_{i}\right), u\left(t_{i}^{+}\right)\right)} n\left(x\left(t_{i}\right)\right),
$$

where $\sigma=-1$, resp. $\sigma=+1$ if $t_{c}$ is a regular crossing time from $K$ to $K_{c}$, resp. from $K_{c}$ into $K$.

- The transversality condition holds true at the terminal time:

$$
p\left(\tau_{f}\right) \in-N_{\operatorname{Viab}_{K}(f)}\left(x\left(\tau_{f}\right)\right) .
$$

This result is a consequence of the hybrid maximum principle [11]. The jump condition on the covector (2.6) follows using that the Hamiltonian is conserved along any extremal trajectory (since the system is autonomous). Hereafter, we say that an extremal $\left(x, p, p_{0}, u\right)$ trajectory is normal if $p_{0} \neq 0$ and is abnormal if $p_{0}=0$. Whenever an extremal trajectory is normal, we can always assume that $p_{0}=-1$. We shall next use this result in order to characterize optimal trajectories for the time crisis problem governed by the Lotka-Volterra system (see Section 5).

\section{Viability kernel for the Lotka-Volterra system}

In the rest of the paper, we shall focus on the Lotka-Volterra prey-predator model:

$$
\left\{\begin{array}{l}
\dot{x}=r x-x y \\
\dot{y}=-m y+x y-u y
\end{array}\right.
$$

where $r>0$ and $m>0$, and $u$ is a measurable control function taking values within the set $\mathcal{U}$ defined as

$$
\mathcal{U}:=\{u:[0,+\infty) \rightarrow[0, \bar{u}] ; u(\cdot) \text { meas. }\}
$$

We set $\mathcal{D}:=\mathbb{R}_{+}^{*} \times \mathbb{R}_{+}^{*}$ which is invariant by (3.1). Our aim is to address the problem of preserving the preys from the predators, maintaining as much as possible their density above a given threshold $\underline{x}>0$, which amounts to have the state belonging to the set

$$
K(\underline{x}):=\{(x, y) \in \mathcal{D} ; x \geq \underline{x}\} .
$$

Although the mathematical analysis of (3.1) (for a constant control $u$ ) predicts that the preys cannot be extinct in finite time, one may consider that practically having a small density of preys expose them to a danger of disappearance that should be avoided as much as possible. This is why, we wish to study in the rest of the paper the minimal time crisis problem governed by $(3.1)$ associated to the set $K(\underline{x})$. We first determine the viability kernel of $K(\underline{x})$ under the dynamics (3.1) and its attainability from the set $\mathcal{D}$. 


\subsection{Computation of the viability hernel}

For a fixed $u \in[0, \bar{u}]$, we define the function $W_{u}: \mathcal{D} \rightarrow \mathbb{R}$ by:

$$
W_{u}(x, y):=x-(m+u) \ln x+y-r \ln y, \quad(x, y) \in \mathcal{D},
$$

together with the number $\underline{c}(u) \in \mathbb{R}$ defined by

$$
\underline{c}(u):=W_{u}(m+u, r)=(m+u)(1-\ln (m+u))+r(1-\ln r),
$$

and the positive equilibrium point $E^{\star}(u)$ for $(3.1)$

$$
E^{\star}(u):=\left(x^{\star}(u), y^{\star}\right)=(m+u, r) .
$$

For a given number $c \geq \underline{c}(u)$, we denote by $L_{u}(c)$, resp. by $S_{u}(c)$, the level set, resp. the sub-level set of $W_{u}$ defined by

$$
L_{u}(c):=\left\{(x, y) \in \mathcal{D}, W_{u}(x, y)=c\right\}, \text { resp. } S_{u}(c):=\left\{(x, y) \in \mathcal{D}, W_{u}(x, y) \leq c\right\} .
$$

We recall in the two following Lemmas classical results about the model (3.1) with constant control.

Lemma 3.1. For a constant control $u$, a trajectory of (3.1) belongs to a level set $L_{u}(c)$ with $c \geq \underline{c}(u)$. The sets $L_{u}(c)$ are closed curves that surround the steady state $E^{\star}(u)$.

Proof. By differentiating $W_{u}$ w.r.t. $x$ and $y$, one finds $\partial_{x} W_{u}(x, y)=1-\frac{m+u}{x}$ and $\partial_{x} W_{u}(x, y)=1-\frac{r}{y}$ for $(x, y) \in \mathcal{D}$. If $(x(\cdot), y(\cdot))$ is a solution of $(3.1)$ with the constant control $u$, a direct computation gives

$$
\frac{d}{d t} W_{u}(x(t), y(t))=\partial_{x} W_{u} \dot{x}+\partial_{y} W_{u} \dot{y}=0 .
$$

So, any solution of (3.1) with the constant control $u$ belongs to a level set of the function $W_{u}$. As $W_{u}(x, y) \rightarrow$ $+\infty$ when $|(x, y)| \rightarrow+\infty$, each level set $L_{u}(c)$ is bounded. For a constant control $u$, one can check that the single equilibrium of the dynamics in $\mathcal{D}$ is $E^{\star}(u)$, and that the level set $L_{u}(\underline{c}(u))$ is the singleton $\left\{E^{\star}(u)\right\}$. Therefore, for any initial condition in $\mathcal{D} \backslash\left\{E^{\star}(u)\right\}$, the trajectory belongs to a level set $L_{u}(c)$ with $c>\underline{c}(u)$ (recall that $W_{u}(x, y) \rightarrow+\infty$ when $\left.|(x, y)| \rightarrow+\infty\right)$. As $L_{u}(c)$ is a compact set that does not contain any equilibrium point, Poincaré-Bendixon Theorem allows to state that the trajectory converges to a limit cycle that belongs to the same level set $L_{u}(c)$. Therefore, the trajectory is periodic and $L_{u}(c)$ is a closed curve which surrounds $E^{\star}(u)$.

For $u \in[0, \bar{u}]$, we define two functions $\phi_{u}: \mathbb{R}_{+} \rightarrow \mathbb{R}$ and $\psi: \mathbb{R}_{+} \rightarrow \mathbb{R}$ by

$$
\phi_{u}(x):=x-(m+u) \ln x, x \in \mathbb{R}_{+} \quad \text { and } \quad \psi(y):=y-r \ln y, y \in \mathbb{R}_{+} .
$$

Lemma 3.2. Given $u \in[0, \bar{u}]$, one has the following properties

(i) For any $x>\phi_{u}(m+u)$, there exists unique $x_{u}^{+}(x) \in(m+u,+\infty)$ and $x_{u}^{-}(x) \in(0, m+u)$ such that $\phi_{u}\left(x_{u}^{+}(x)\right)=\phi_{u}\left(x_{u}^{-}(x)\right)=x$.

(ii) If $p>\psi(r)$, the equation $\psi(y)=p$ has exactly two roots $y^{-}(p), y^{+}(p)$ that satisfy $y^{-}(p)<r<y^{+}(p)$.

Proof. One can easily check that $\lim _{x \rightarrow+\infty} \phi_{u}(x)=\lim _{x \rightarrow 0} \phi_{u}(x)=+\infty$. Moreover, by differentiating $\phi_{u}$ w.r.t. $x$, one finds $\phi_{u}^{\prime}(x)=1-\frac{m+u}{x}$. So the function $\phi_{u}$ is decreasing from $+\infty$ down to $\phi_{u}(m+u)$ and increasing up to $+\infty$. Therefore, for any $x>\phi_{u}(m+u)$, the equation $\phi_{u}(z)=x$ has exactly two solutions $x_{u}^{-}(x), x_{u}^{+}(x)$, with $x_{u}^{-}(x)<m+u$ and $x_{u}^{+}(x)>m+u$ which proves (i). Similarly, the function $\psi$ is decreasing from $+\infty$ down to $\psi(r)$ and increasing up to $+\infty$, which gives (ii).

For $c \in \mathbb{R}$, we consider the subsets of $\mathcal{D}, L_{u}^{+}(c), L_{u}^{-}(c), S_{u}^{+}(c)$ and $S_{u}^{-}(c)$ defined by:

$$
L_{u}^{+}(c):=L_{u}(c) \cap\{y \geq r\}, \quad L_{u}^{-}(c):=L_{u}(c) \cap\{y \leq r\},
$$

and

$$
S_{u}^{+}(c):=S_{u}(c) \cap\{y \geq r\}, \quad S_{u}^{-}(c):=S_{u}(c) \cap\{y \leq r\},
$$

and let $r_{-} \in(0, r]$ be defined by:

$$
r_{-}:=y^{-}\left(W_{0}\left(x_{\bar{u}}^{+}(\underline{x}), r\right)-\phi_{0}(\underline{x})\right) .
$$

The next Proposition provides a description of the viability kernel $\operatorname{Viab}(\underline{x})$ of $K(\underline{x})$ for $(3.1)$. 
Proposition 3.1. One has the following characterization of the viability kernel:

(i) If $m+\bar{u}<\underline{x}$, the set $\operatorname{Viab}(\underline{x})$ is empty.

(ii) If $\bar{u} \geq \underline{x}-m$, the viability kernel is non-empty and is given by

$$
\operatorname{Viab}(\underline{x})=S_{\bar{u}}^{+}\left(W_{\bar{u}}(\underline{x}, r)\right) \bigcup\left(S_{0}^{-}\left(W_{0}\left(x_{\bar{u}}^{+}(\underline{x}), r\right)\right) \cap K(\underline{x})\right),
$$

where $x_{\bar{u}}^{+}(\underline{x})$ is given by Lemma 3.2. Its boundary is the union of the three curves

$$
\begin{aligned}
B^{+}(\underline{x}) & :=L_{\bar{u}}^{+}\left(W_{\bar{u}}(\underline{x}, r)\right), \\
B^{-}(\underline{x}) & :=L_{0}^{-}\left(W_{0}\left(x_{\bar{u}}^{+}(\underline{x}), r\right)\right) \cap\{x \geq \underline{x}\}, \\
B^{0}(\underline{x}) & :=\{\underline{x}\} \times\left[r_{-}, r\right] .
\end{aligned}
$$

Proof. Let us assume that $\bar{u}<\underline{x}-m$ and let $\varepsilon>0$ be such that $m+\underline{u}<\underline{x}-\varepsilon$. Consider a trajectory $(x(\cdot), y(\cdot))$ that stays in the set $K(\underline{x})$ for any time $t \geq 0$. As $0 \leq u(t) \leq \bar{u}$, we deduce that

$$
\dot{y}=y(x-m-u) \geq y(x-\underline{x}+\varepsilon) \geq \varepsilon y,
$$

using that $x(t) \geq \underline{x}$ for any time $t \geq 0$. Therefore $y(\cdot)$ is increasing, unbounded and thus there exists $t_{1}>0$ such that $y(t)>r$ for any time $t \geq t_{1}$. It follows that

$$
\dot{x}(t)=x(t)(r-y(t))<x(t)\left(r-y\left(t_{1}\right)\right) \quad \forall t>t_{1},
$$

implying that for $t>t_{1}$ one has $\dot{x}(t)<-C x(t)$ with $C:=y\left(t_{1}\right)-r>0$. Thus, there exists $t_{2}>t_{1}$ such that $x\left(t_{2}\right)<\underline{x}$. So the trajectory $(x(\cdot), y(\cdot))$ must escape the set $K(\underline{x})$ in finite time, and we have a contradiction. Thus, the viability kernel $\operatorname{Viab}(\underline{x})$ is empty which proves (i).

Assume now that one has $\bar{u} \geq \underline{x}-m$ and let us prove (ii). Notice first that the three curves $B^{+}(\underline{x}), B^{-}(\underline{x})$ and $B^{0}(\underline{x})$ belong to the set $K(\underline{x})$ and that their union $U(\underline{x})$ defines the boundary of a compact subset $Z(\underline{x})$ of $K(\underline{x})$, which is such that

$$
Z(\underline{x})=S_{\bar{u}}^{+}\left(W_{\bar{u}}(\underline{x}, r)\right) \bigcup\left(S_{0}^{-}\left(W_{0}\left(x_{\bar{u}}^{+}(\underline{x}), r\right)\right) \cap K(\underline{x})\right) .
$$

When $\bar{u}=\underline{x}-m$, the set $Z(\underline{x})$ is reduced to the single point $E^{\star}(\bar{u})$ that is an equilibrium of $(3.1)$ for the constant control $\bar{u}$. Thus, $Z(\underline{x})$ is a viable set.

When $\bar{u}>\underline{x}-m$, we first show that for any initial condition in $U(\underline{x})$, there exists a trajectory that stays in $K(\underline{x})$ for any time $t \geq 0$. Consider an initial condition in the set $B^{+}(\underline{x})$. With the control $u=\bar{u}$, the corresponding solution of (3.1) remains on the level set $L_{\bar{u}}\left(W_{\bar{u}}(\underline{x}, r)\right)$ which is contained in $K(\underline{x})$ as its extreme left point is $(\underline{x}, r)$. Take now an initial condition in $B^{-}(\underline{x})$. With the control $u=0$, the corresponding solution of $(3.1)$ remains on $B^{-}(\underline{x})$ until it reaches in finite time the boundary point $\left(x_{\bar{u}}^{+}(\underline{x}), r\right)$ that belongs to $B^{+}(\underline{x})$. From this point, we come back to the previous case. Finally, take an initial condition in $\operatorname{Int}\left(B^{0}(\underline{x})\right)($ if not empty). On $\operatorname{Int}\left(B^{0}(\underline{x})\right)$, one has $\dot{x}>0$ for any control as one has $r-y>0$. Thus the trajectory enters the subset $Z(\underline{x})$ and cannot evade from $K(\underline{x})$ on $\operatorname{Int}\left(B^{0}(\underline{x})\right)$. If the trajectory touches $B^{+} \cup B^{-}$, we face one of the two previous cases. So we conclude that $Z(\underline{x})$ is a viable domain.

We now show that $Z(\underline{x})$ is the largest viable domain included in $K(\underline{x})$, that is, the viability kernel $V i a b(\underline{x})$, or equivalently that any trajectory with initial condition in $K(\underline{x}) \backslash Z(\underline{x})$ leaves the set $K(\underline{x})$ in a finite horizon. For convenience we consider the two subsets of $K(\underline{x}) \backslash Z(\underline{x}), C^{+}(\underline{x})$ and $C^{-}(\underline{x})$ defined by:

$$
C^{+}(\underline{x})=(K(\underline{x}) \backslash Z(\underline{x})) \cap\{y \geq r\} \quad \text { and } \quad C^{-}(\underline{x})=(K(\underline{x}) \backslash Z(\underline{x})) \cap\{y \leq r\} .
$$

Consider now an initial condition $\left(x_{0}, y_{0}\right) \in C^{+}(\underline{x})$, and let $(x(\cdot), y(\cdot))$ a solution of $(3.1)$ starting from $\left(x_{0}, y_{0}\right)$. One has $W_{\bar{u}}\left(x_{0}, y_{0}\right)>W_{\bar{u}}(\bar{x}, r)$, and by differentiating w.r.t $t$ one finds:

$$
\frac{d}{d t} W_{\bar{u}}(x(t), y(t))=(y(t)-r)(\bar{u}-u(t)) \geq 0 .
$$

Therefore no trajectory can reach the level set $L_{\bar{u}}\left(W_{\bar{u}}(\bar{x}, r)\right)$ from $K(\underline{x}) \backslash Z(\underline{x})$. When $y(t)=r$, one has $x(t)>m+\bar{u}$ and thus $\dot{y}(t)=r(x(t)-m-u(t))>0$ as $u(t) \leq \bar{u}$. We deduce that if there exists a trajectory 
with an initial condition $\left(x_{0}, y_{0}\right)$ in $C^{+}(\underline{x})$ that stays in $K(\underline{x})$, it has to stay in $C^{+}(\underline{x})$. By differentiating w.r.t $t$, we obtain on $C^{+}(\underline{x})$ that

$$
\frac{d}{d t} W_{0}(x(t), y(t))=-u(t)(y(t)-r) \leq 0,
$$

and thus $W_{0}(x(t), y(t)) \leq W_{0}\left(x_{0}, y_{0}\right)$. It follows that the trajectory is bounded. Moreover, one has $\dot{x} \leq 0$. $i . e$. the function $t \mapsto x(t)$ is non-increasing and thus converges to a certain $x_{\infty}>0$. By Barbalat's Lemma (see for instance [24]), $\dot{x}(t)$ converges to 0 which implies that $y(t)$ tends to $r$. Then $W_{\bar{u}}(x(t), y(t))$ converges to $W_{\bar{u}}\left(x_{\infty}, r\right)>W_{\bar{u}}(\bar{x}, r)$ which implies that $x_{\infty}<\bar{x}$. Thus, the trajectory necessarily leaves the set $K(\underline{x})$ and we have a contradiction.

Consider now an initial condition $\left(x_{0}, y_{0}\right) \in C^{-}(\underline{x})$. Similarly, one can show that no trajectory can reach the level set $L_{0}\left(W_{0}\left(x^{+}(\underline{x}), r\right)\right)$ from $K(\underline{x}) \backslash Z(\underline{x})$. It follows that a trajectory with an initial condition in $C^{-}(\underline{x})$ that stays in $K(\underline{x})$ has to stay in $C^{-}(\underline{x})$ (otherwise, it reaches $C^{+}(\underline{x})$ and we have shown above that its has to escape $K(\underline{x})$ ). As previously, one can show that a trajectory that stays in $C^{-}(\underline{x})$ is bounded and as $t \mapsto x(t)$ is increasing, one obtains the convergence of $x(t)$ to a certain $x_{\infty}>\underline{x}$. As before, by Barbalat's Lemma, $\dot{x}(t)$ converges to 0 when $t \rightarrow+\infty$, which implies that $y(t) \rightarrow r$, and thus $x_{\infty} \geq x_{\bar{u}}^{+}(\underline{x})>m+\bar{u}$. Therefore there exists $\varepsilon>0$ and $t_{0}>0$ such that $\dot{y}(t)=(x(t)-m-u) y(t)>\varepsilon y(t)$ for any $t>t_{0}$. This gives a contradiction with the convergence of $y(t)$ to $r$ when $t \rightarrow+\infty$. So, the trajectory has to enter $C^{+}(\underline{x})$ and then leaves $K(\underline{x})$. This ends the proof of (ii).

The viability kernel is depicted on Fig. 1 in case (ii) of Proposition 3.1 together with the three curves $B^{+}(\underline{x}), B^{-}(\underline{x}), B^{0}(\underline{x})$ that define its boundary (see the Appendix for the numerical values of the parameters). It is worth noting that $B^{+}(\underline{x})$ is a semi-orbit of $(3.1)$ with $u=\bar{u}$ passing trough the point $(\underline{x}, r)$. Similarly, $B^{-}(\underline{x})$ is a semi-orbit of $(3.1)$ with $u=0$ passing though the point $\left(\underline{x}, r_{-}\right)$. Note that whenever $m+\bar{u}=\underline{x}$, then the viability kernel is reduced to one point i.e.

$$
m+\bar{u}=\underline{x} \quad \Rightarrow \quad \operatorname{Viab}(\underline{x})=\{(\underline{x}, r)\},
$$

as the semi-orbit $B^{+}(\underline{x})$ is then reduced to the positive equilibrium point of $(3.1)$ with $u=\bar{u}$. The viability

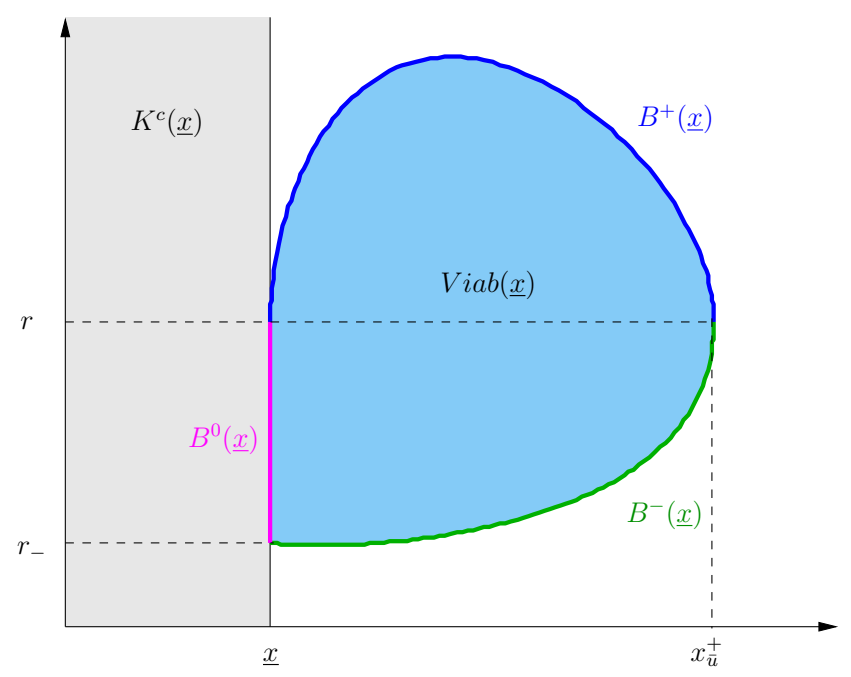

Figure 1: Viability kernel when $\bar{u}>\underline{x}-m$ (numerical values can be found in the appendix).

kernel of $K(\underline{x})$ enjoys the following properties.

Proposition 3.2. Suppose that $\bar{u}>\underline{x}-m$.

(i) Consider the unique solution of (3.1) backward in time with $u=0$ from $\left(x_{\bar{u}}^{+}(\underline{x}), r\right)$, and let $t^{\prime}>0$ be the first time where this trajectory intersects the line $\{y=r\}$. Then, we have:

$$
x\left(t^{\prime}\right) \leq \underline{x}
$$


(ii) The set $\operatorname{Viab}(\underline{x})$ is a compact convex set with non-empty interior.

(iii) Suppose that $t_{1}<t_{2}$ are two consecutive crossing times from $K(\underline{x})$ to $K(\underline{x})^{c}$ and from $K(\underline{x})^{c}$ to $K(\underline{x})$ respectively, that $\left(x\left(t_{1}\right), y\left(t_{1}\right)\right) \notin V i a b(\underline{x})$, and that $\left(x\left(t_{2}\right), y\left(t_{2}\right)\right) \notin V i a b(\underline{x})$. Then, one has:

$$
t_{2}-t_{1} \geq \frac{\ln \left(\frac{r}{r_{-}}\right)}{m+\bar{u}}
$$

Proof. To prove (i), suppose by contradiction that $x\left(t^{\prime}\right)>\underline{x}$. Denote by $\left(x_{0}(\cdot), x_{0}(\cdot)\right)$ the unique solution of (3.1) with $u=0$ and such that $\left(x_{0}(0), x_{0}(0)\right)=\left(x\left(t^{\prime}\right), r\right)$. By construction, the point $\left(x_{\bar{u}}^{+}(\underline{x}), r\right)$ is on the graph of the parameterized curve $\left(x_{0}(\cdot), y_{0}(\cdot)\right)$. Thus we denote by $t_{0}:=\inf \left\{t \geq 0 ;\left(x_{0}(t), x_{0}(t)\right)=\left(x_{\bar{u}}^{+}(\underline{x}), r\right)\right\}$, and let $\gamma_{0}$ be the parametrized curve $\left(x_{0}(\cdot), x_{0}(\cdot)\right)$ on the interval $\left[0, t_{0}\right]$.

Now, consider the unique solution $\left(x_{1}(\cdot), y_{1}(\cdot)\right)$ of $(3.1)$ with $u=\bar{u}$ starting from the point $\left(x\left(t^{\prime}\right), r\right)$ and let $t_{1}:=\inf \left\{t \geq 0 ; y_{1}(t)=r\right\}$. We define $\gamma_{1}$ as the parametrized curve $\left(x_{1}(\cdot), y_{1}(\cdot)\right)$ on the interval $\left[0, t_{1}\right]$. From (3.1), we know that the graph of $\gamma_{1}$ is below the graph of $\gamma_{0}$. To conclude, we consider the unique solution $\left(\tilde{x}_{1}(\cdot), \tilde{y}_{1}(\cdot)\right)$ of $(3.1)$ with $u=\bar{u}$ starting from $(\underline{x}, r)$ until the first time $t_{1}^{\prime}>0$ where it reaches the segment line $\{y=r\}$. It can be noticed that this curve passes through the point $\left(x_{\bar{u}}^{+}(\underline{x}), r\right)$ and that its graph $\tilde{\gamma}_{1}$ is also below the graph of $\gamma_{0}$. Hence, both graphs $\gamma_{1}$ and $\tilde{\gamma}_{1}$ should intersect i.e. there must exist a time $\tau \in\left(0, t_{0}\right)$ such that $\left(x_{1}(\tau), y_{1}(\tau)\right)=\left(\tilde{x}_{1}(\tau), \tilde{y}_{1}(\tau)\right)$. By Cauchy-Lipschitz's Theorem, both solutions $\left(x_{1}(\cdot), y_{1}(\cdot)\right)$ and $\left(\tilde{x}_{1}(\cdot), \tilde{y}_{1}(\cdot)\right)$ should coincide everywhere which is a contradiction as $\left(x_{1}(0), y_{1}(0)\right) \neq\left(\tilde{x}_{1}(0), \tilde{y}_{1}(0)\right)$.

Let us now show (ii). From Proposition 3.1 and the previous point, we know that $\operatorname{Viab}(\underline{x})$ is a compact subset of $\mathbb{R}^{2}$ with non-empty interior. Now, one can easily verify that solutions of $(3.1)$ in the plane $(x, y)$ satisfy:

$$
\left.\frac{d^{2} y}{d x^{2}}\right|_{u=0}(x)=\frac{y\left(r(x-m)^{2}+m(r-y)^{2}\right)}{(r-y)^{3} x^{2}} \quad \text { and }\left.\quad \frac{d^{2} y}{d x^{2}}\right|_{u=\bar{u}}(x)=\frac{y\left(r(x-m-1)^{2}+(m+1)(r-y)^{2}\right)}{(r-y)^{3} x^{2}}
$$

It follows that for $y<r$, resp. $y>r$, one has $\left.\frac{d^{2} y}{d x^{2}}\right|_{u=0}(x)>0$, resp. $\left.\frac{d^{2} y}{d x^{2}}\right|_{u=\bar{u}}(x)<0$, which guarantees that the set $\operatorname{Viab}(\underline{x})$ is convex.

Finally, to prove (iii), we integrate the equation $\dot{y}(t)=y(t)(x(t)-m-u(t))$ over $\left[t_{1}, t_{2}\right]$, which gives:

$$
(m+\bar{u})\left(t_{2}-t_{1}\right) \geq \int_{t_{1}}^{t_{2}}(m+u(t)) \mathrm{d} t=\int_{t_{1}}^{t_{2}} x(t) \mathrm{d} t-\int_{y\left(t_{1}\right)}^{y\left(t_{2}\right)} \frac{\mathrm{d} y}{y} \geq-\int_{y\left(t_{1}\right)}^{y\left(t_{2}\right)} \frac{\mathrm{d} y}{y} \geq \ln \left(\frac{r}{r_{-}}\right),
$$

using that $y\left(t_{1}\right)>r$ and $y\left(t_{2}\right)<r_{-}$. This ends the proof.

Remark 3.1. (i) Whereas when $m<\underline{x}$, it is clear that (3.2) holds true (as the equilibrium point for (3.1) with $u=0$ is $(m, r)$ ), the previous Proposition shows that this property remains valid whenever $m>\underline{x}$. Note also that in the latter case, the set Viab(x) is always non-empty as $\bar{u}>0$.

(ii) We know that for a given initial state in $\operatorname{Viab}(\underline{x})$, any control $u$ can be chosen until that the corresponding trajectory reaches the boundary of $\operatorname{Viab}(\underline{x})$ (see e.g. $[2,3])$. If $\left(x_{0}, y_{0}\right) \in B^{-}(\underline{x})$, resp. $\left(x_{0}, y_{0}\right) \in B^{+}(\underline{x})$, then only the control $u=0$, resp. $u=\bar{u}$ is admissible in order to stay in Viab( $\underline{x})$.

(iii) If $\underline{x}>m$, then any point of the segment $[\underline{x}, \bar{u}+m] \times\{r\}$ is a steady-state point for (3.1) with a prescribed constant control whereas if $\underline{x} \leq m$, then any point of the segment $[m, \bar{u}+m] \times\{r\}$ is a steady-state point for (3.1) with a prescribed constant control.

\subsection{Attainability of the viability kernel}

In order to show the attainability of the target set, it is convenient to introduce the following feedback control:

$$
\mathbf{u}[x, y]:= \begin{cases}\bar{u} & \text { if } \quad y \geq r \\ 0 & \text { if } \quad y<r .\end{cases}
$$

Given an initial condition $\left(x_{0}, y_{0}\right) \in\left(\mathbb{R}_{+}^{*} \times \mathbb{R}_{+}^{*}\right) \backslash V i a b(\underline{x})$, we denote by $\left(x_{m}(\cdot), y_{m}(\cdot)\right)$ the unique solution of (3.1) starting from $\left(x_{0}, y_{0}\right)$ at time 0 associated to the control $u_{m}(\cdot)$ defined by $u_{m}(t):=\mathbf{u}\left[x_{m}(t), y_{m}(t)\right]$. 
Proposition 3.3. For any initial condition $\left(x_{0}, y_{0}\right) \in \mathcal{D} \backslash$ Viab $(\underline{x})$, there exists a control $u \in \mathcal{U}$ steering $\left(x_{0}, y_{0}\right)$ to the viability kernel Viab( $\underline{x})$.

Proof. Suppose first that one has $m+\bar{u}>\underline{x}$, hence $\operatorname{Viab}(\underline{x})$ has a non-empty interior.

First step. We show that it is enough to prove the result for any initial condition of type $\left(x_{0}, r\right)$ with $x_{0}>x_{\bar{u}}^{+}(\bar{x})$. If the initial condition $\left(x_{0}, y_{0}\right)$ is such that $x_{0}<r$, then it is enough to replace $x_{0}$ by $x_{m}\left(t_{c}\right)$ where $t_{c}$ is the first time $t>0$ such that $y_{m}\left(t_{c}\right)=r$. If $\left(x_{m}\left(t_{c}\right), y_{m}\left(t_{c}\right)\right) \in \operatorname{Viab}(\underline{x})$, then the result is proved. Otherwise, we have $x_{m}\left(t_{c}\right)>x_{\bar{u}}^{+}(\bar{x})$. If now $x_{0}>r$, we apply the control $u_{m}$ until the first time $t_{c}^{\prime}>0$ such that $y_{m}\left(t_{c}^{\prime}\right)=r$. Then, for $t>t_{c}^{\prime}\left(\right.$ close to $\left.t_{c}^{\prime}\right)$ one has $y_{m}\left(t_{c}^{\prime}\right)<r$, and we conclude by the previous case.

Second step. We now show the Proposition for any initial condition $\left(x_{0}, r\right)$ with $x_{0}>x_{\bar{u}}^{+}(\bar{x})$. By applying the feedback control $u_{m}$, we can define two sequences of time $\left(t_{n}\right)_{n \geq 0}$ and $\left(t_{n}^{\prime}\right)_{n \geq 0}$ such that:

$$
y_{m}\left(t_{n}\right)=y_{m}\left(t_{n}^{\prime}\right)=r \quad \text { and } \quad x_{n}^{\prime}:=x_{m}\left(t_{n}^{\prime}\right)<\underline{x}<x_{n}:=x_{m}\left(t_{n}\right) .
$$

Moreover, the trajectory is such that for any $n \in \mathbb{N}$ :

$$
t \in\left(t_{n}, t_{n}^{\prime}\right) \Rightarrow y_{m}(t)>r \quad \text { and } \quad t \in\left(t_{n}^{\prime}, t_{n+1}\right) \Rightarrow y_{m}(t)<r .
$$

We have $x_{1}<x_{0}$. Indeed, consider the two solutions of $(3.1), \hat{x}_{0}(\cdot)$, resp. $\hat{x}_{1}(\cdot)$ with the control $u=0$, resp. $u=\bar{u}$ starting from the point $\left(x_{0}^{\prime}, r\right)$. We then have $\hat{x}_{0}(t)>\hat{x}_{1}(t)$ for any $t \in(0, \hat{t}]$ where $\hat{t}$ is such that $\hat{x}_{0}(\hat{t})=r$. Now, as $\hat{x}_{1}(\cdot)$ passes though the point $\left(x_{0}, r\right)$, we deduce that $x_{1}<x_{0}$. Now, the two solutions of (3.1) with $u=\bar{u}$ starting from $\left(x_{0}, r\right)$ and $\left(x_{1}, r\right)$ cannot intersect, thus we deduce that $x_{1}^{\prime}>x_{0}^{\prime}$. By induction, we obtain that $\left(x_{n}\right)_{n \geq 0}$ is decreasing and that $\left(x_{n}^{\prime}\right)_{n \geq 0}$ is increasing.

Now, integrating $(3.1)$ on the interval $\left(t_{0}, t_{0}^{\prime}\right)$, resp. $\left(t_{0}^{\prime}, t_{1}\right)$ with $u=\bar{u}$, resp. with $u=0$ yields:

$$
\left\{\begin{array}{l}
-x_{0}+(m+\bar{u}) \ln \left(x_{0}\right)=-x_{0}^{\prime}+(m+\bar{u}) \ln \left(x_{0}^{\prime}\right) \\
-x_{1}+m \ln x_{1}=-x_{0}^{\prime}+m \ln x_{0}^{\prime}
\end{array}\right.
$$

Thus we obtain the relation $x_{0}-x_{1}-m \ln \left(\frac{x_{0}}{x_{1}}\right)+\bar{u} \ln \left(\frac{x_{0}^{\prime}}{x_{0}}\right)=0$ and by induction we get:

$$
\forall n \in \mathbb{N}^{*}, \quad x_{n-1}-x_{n}-m \ln \left(\frac{x_{n-1}}{x_{n}}\right)+\bar{u} \ln \left(\frac{x_{n-1}^{\prime}}{x_{n-1}}\right)=0 .
$$

As $x_{n-1}<x_{n}$, we deduce that one has

$$
x_{n-1}-x_{n} \geq \bar{u} \ln \left(\frac{x_{n-1}}{x_{n-1}^{\prime}}\right) .
$$

To conclude, we suppose by contradiction that the trajectory always stays outside the set $V i a b(\underline{x})$. By noticing that inequalities $x_{n-1}-x_{n-1}^{\prime} \geq x_{\bar{u}}^{+}(\underline{x})-\underline{x}$ and $x_{n-1}^{\prime} \leq \underline{x}$ are fulfilled for any $n \geq 1$, one obtains $\frac{x_{n-1}}{x_{n-1}^{\prime}} \geq \frac{x_{\bar{u}}^{+}(\bar{x})}{\underline{x}}$ which implies

$$
x_{n-1}-x_{n} \geq \beta
$$

where $\beta:=\bar{u} \ln \left(\frac{x_{\bar{u}}^{+}(\bar{x})}{\underline{x}}\right)>0$ (recall that the interior of $V i a b(\underline{x})$ is non-empty). Thus, one has for each $n \in \mathbb{N}$ $x_{n} \leq x_{n-1}-\beta$. Therefore we obtain a contradiction and the trajectory necessary enters the set $\operatorname{Viab}(\underline{x})$ which ends the proof in the case where $m+\bar{u}>\underline{x}$.

Consider now the case $m+\bar{u}=\underline{x}$. Then $\operatorname{Viab}(\underline{x})$ is reduced to a single point $(\underline{x}, r)$ that belongs to the periodic orbit $O_{r}$ defined as the unique solution of (3.1) with $u=0$ passing through this point. The second intersection point of this orbit with the line $\{y=r\}$ is denoted by $(\nu, r)$ with $0<\nu<\underline{x}$. Now, if the initial condition is in the interior of $O_{r}$, then the control $u=\bar{u}$ steers (3.1) in finite time to $O_{r}$ and the result follows. Following the proof of the result in the case where $m+\bar{u}>\underline{x}$, we can suppose that the initial condition $\left(x_{0}, r\right)$ is such that $x_{0}>\underline{x}$. Similarly, let us define two sequences of points $\left(x_{n}\right),\left(x_{n}^{\prime}\right)$ such that $\left(x_{n}\right)$ is increasing and $\left(x_{n}^{\prime}\right)$ is decreasing. Moreover inequality (3.5) also holds true. To conclude, we suppose by contradiction that the trajectory does not intersect $O_{r}$. Hence, for any $n \in \mathbb{N}$ one has $x_{n}-x_{n}^{\prime} \geq \underline{x}-\nu$ and $x_{n}^{\prime} \leq \nu$. We then find that for any $n \in \mathbb{N}^{*}$

$$
\frac{x_{n-1}}{x_{n-1}^{\prime}}=\frac{x_{n-1}-x_{n-1}^{\prime}}{x_{n-1}^{\prime}}+1 \geq \frac{x}{\nu}>0,
$$


and for any $n \in \mathbb{N}$ we obtain

$$
x_{n-1}-x_{n} \geq \beta^{\prime},
$$

with $\beta^{\prime}:=\bar{u} \ln (\underline{x} / \nu)>0$. We can then conclude as in the previous case.

\section{Minimal time problem to reach the viability kernel}

In this Section, we consider that the condition $\bar{u} \geq \underline{x}-m$ is fulfilled, which guarantees that the viability kernel $\operatorname{Viab}(\underline{x})$ is non empty (see Proposition 3.1). We compute the optimal synthesis for the minimal time control problem to reach $\operatorname{Viab}(\underline{x})$. The value function $V$ associated to the minimum time control problem to reach $\operatorname{Viab}(\underline{x})$ is defined as follows. For a given initial condition $z_{0}=\left(x_{0}, y_{0}\right) \in \mathcal{D}$, the function $V$ is defined as

$$
V\left(z_{0}\right):=\inf _{u \in \mathcal{U}} T_{u} \quad \text { s.t. } z\left(T_{u}, z_{0}\right) \in \operatorname{Viab}(\underline{x})
$$

where $z\left(\cdot, z_{0}\right):=(x, y)$ is the unique solution of (3.1) associated to the control $u \in \mathcal{U}$ and $T_{u}$ the first entry time of $z\left(\cdot, z_{0}\right)$ into the target set. From Proposition 3.3, the set $\operatorname{Viab}(\underline{x})$ can be reached from any initial condition $\left(x_{0}, y_{0}\right) \in \mathcal{D}$. Therefore $V$ is finite everywhere in $\mathcal{D}$ and the existence of an optimal control follows from standard argumentation based on Filipov's Theorem (see for instance [10]). Recall also that $V i a b(\underline{x})$ can be reached from $K(\underline{x})^{c}$ only through $\partial K(\underline{x}) \backslash \partial V i a b(\underline{x})$ (see for instance [2]), that is here the line-segment $B^{0}(\underline{x})$. Let $H: \mathbb{R}^{2} \times \mathbb{R}^{2} \times \mathbb{R} \times \mathbb{R} \rightarrow \mathbb{R}$ be the Hamiltonian associated to (4.1) defined by:

$$
H=H\left(x, y, p, q, p_{0}, u\right)=p x(r-y)+q y(x-m-u)+p_{0} .
$$

We now apply the Pontryagin Maximum Principle (PMP) to (4.1) to derive necessary optimality conditions for Problem (4.1). Let $u \in \mathcal{U}$ be an optimal control defined over a certain time interval $\left[0, T_{u}\right]$ with $T_{u}<+\infty$ and let $z:=(x, y)$ be the associated trajectory. Then, there exists an absolutely continuous map $\lambda:=(p, q)$ : $\left[0, T_{u}\right] \rightarrow \mathbb{R}^{2}$ and a number $p_{0} \leq 0$ such that the following conditions are satisfied:

- The pair $\left(\lambda(\cdot), p_{0}\right)$ is non-zero.

- The adjoint vector satisfies the adjoint equation $\dot{\lambda}=-\frac{\partial H}{\partial z}$ a.e., that is:

$$
\left\{\begin{array}{l}
\dot{p}=p(y-r)-q y \\
\dot{q}=p x+q(u+m-x)
\end{array}\right.
$$

- The transversality condition can be expressed as $\lambda\left(T_{u}\right) \in-N_{\operatorname{Viab}(\underline{x})}\left(z\left(T_{u}\right)\right)$.

- The Hamiltonian condition reeds as follows:

$$
u(t) \in \arg \max _{0 \leq \omega \leq \bar{u}} H\left(z(t), \lambda(t), p_{0}, \omega\right) \quad \text { a.e. } t \in\left[0, T_{u}\right]
$$

An extremal is a triplet $(z, \lambda(\cdot), u)$ satisfying (3.1)-(4.2)-(4.3). Moreover, as the system is autonomous and $T_{u}$ is free, the Hamiltonian is equal to zero along any extremal trajectory. In view of the maximization condition in (4.3), we define the switching function $\phi$ as

$$
\phi:=-q y
$$

and we obtain the following optimal control law:

$$
\left\{\begin{array}{l}
\phi(t)>0 \quad \Rightarrow \quad u(t)=\bar{u}, \\
\phi(t)<0 \quad \Rightarrow \quad u(t)=0, \\
\phi(t)=0 \quad \Rightarrow \quad u(t) \in[0, \bar{u}] .
\end{array}\right.
$$

We call switching time $t_{c}$ a time such that the switching function $\phi$ has non-constant sign in any neighborhood of $t_{c}$ (and switching point for the corresponding state $z\left(t_{c}\right)$ ). From (4.4), we deduce that any switching time satisfies $\phi\left(t_{c}\right)=0$. A direct computation shows that we have:

$$
\dot{\phi}(t)=-p(t) x(t) y(t) \quad \text { a.e. } t \in\left[0, T_{u}\right]
$$


Let $e_{1}:=(1,0)$. Since $\operatorname{Viab}(\underline{x})$ is non-smooth at the point $\left(\underline{x}, r_{-}\right)$, let $w$ be the unit vector defined by $w:=(\sin \psi,-\cos \psi)$ where $\psi \in\left(-\frac{\pi}{2}, \frac{\pi}{2}\right)$ is defined by

$$
\tan \psi:=\frac{(\underline{x}-m) r_{-}}{\left(r-r_{-}\right) \underline{x}}
$$

Lemma 4.1. Suppose that $\bar{u}>\underline{x}-m$, i.e. that $V i a b(\underline{x})$ has a non-empty interior. If $(x, y) \in B^{0}(\underline{x})$, we have:

$$
\begin{array}{ll}
y \in\left(r_{-}, r\right] \quad \Rightarrow N_{\operatorname{Viab}(\underline{x})}(x, y)=\mathbb{R}_{-} \times\{0\} \\
y=r_{-} \quad \Rightarrow N_{\operatorname{Viab}(\underline{x})}(x, y)=\left\{\alpha\left(\beta w-[1-\beta] e_{1}\right) ;(\alpha, \beta) \in \mathbb{R}_{+} \times[0,1]\right\} .
\end{array}
$$

Proof. The result is straightforward for $y \in\left(r_{-}, r\right]$ (note that for $y=r$, then $B^{+}(\underline{x})$ has a vertical tangent at the point $(\underline{x}, r))$.

Now, at the boundary point $\left(\underline{x}, r_{-}\right)$of $\operatorname{Viab}(\underline{x})$, the tangent cone is generated by the vectors $(0,1)$ and $(\cos \psi, \sin \psi)$. The geometric computation of $N_{\operatorname{Viab}(\underline{x})}(x, y)$ follows using that $\operatorname{Viab}(\underline{x})$ is convex and that the normal cone to $\operatorname{Viab}(\underline{x})$ at $(x, y) \in B^{0}(\underline{x})$ is the dual cone to the tangent cone to $\operatorname{Viab}(\underline{x})$ at $(x, y)$.

Thanks to Pontryagin Principle, we can derive the following properties.

Proposition 4.1. Let $u \in \mathcal{U}$ be an optimal control for (4.1) and $(z, \lambda(\cdot)$, $u)$ the corresponding extremal trajectory defined over a time interval $\left[0, T_{u}\right]$. Then, the following properties hold true:

(i) The control $u$ is bang-bang i.e. it satisfies $u(t) \in\{0, \bar{u}\}$ for a.e. $t \in\left[0, T_{u}\right]$ and:

$$
u(t)=\frac{\bar{u}}{2}(1+\operatorname{sign}(\phi(t))) \quad \text { a.e. } t \in\left[0, T_{u}\right]
$$

(ii) The transversality condition on the adjoint vector at time $T_{u}$ reads as follows (in the case where $\bar{u}>\underline{x}-m$ only):

$$
\begin{array}{lll}
\left(x\left(T_{u}\right), y\left(T_{u}\right)\right) \in\{\underline{x}\} \times\left(r_{-}, r\right) & \Rightarrow \quad\left(p\left(T_{u}\right), q\left(T_{u}\right)\right) \in \mathbb{R}_{+} \times\{0\} \\
\left(x\left(T_{u}\right), y\left(T_{u}\right)\right)=\left(\underline{x}, r_{-}\right) & \Rightarrow \quad\left(p\left(T_{u}\right), q\left(T_{u}\right)\right) \in\left\{\alpha\left(-\beta w+(1-\beta) e_{1}\right) ;(\alpha, \beta) \in \mathbb{R}_{+} \times[0,1]\right\} .
\end{array}
$$

(iii) If the extremal trajectory reaches the target at some point in $\{\underline{x}\} \times\left(r_{-}, r\right)$, then it is normal i.e. $p_{0} \neq 0$.

(iv) If the extremal trajectory is abnormal, then any switching point lies on the line $\{y=r\}$.

Proof. To prove (i), suppose that $\phi=0$ on some time interval $\left[t_{1}, t_{2}\right]$. By differentiating $\phi$ w.r.t the time $t$, we obtain $\dot{q}=q=0$ over $\left[t_{1}, t_{2}\right]$ implying $p=0$ over $\left[t_{1}, t_{2}\right]$. From $(4.2)$, we deduce that the adjoint vector $\lambda$ is zero over $\left[0, T_{u}\right]$. We thus obtain a contradiction with the PMP using $H=0$. This proves that (4.5) holds almost everywhere.

Lemma 4.1 together with the transversality condition $\lambda\left(T_{u}\right) \in-N_{V i a b(\underline{x})}\left(z\left(T_{u}\right)\right)$ straightforwardly implies (4.6) which proves (ii).

Let us now show (iii). Suppose by contradiction that $p_{0}=0$. Using that $H=0$ and that $y\left(T_{u}\right) \neq r$, one obtains $p\left(T_{u}\right)=0$. Thus we would have $p\left(T_{u}\right)=q\left(T_{u}\right)=0$ and then $\lambda \equiv 0$ using (4.2). This contradicts the PMP as the pair $\left(\lambda(\cdot), p_{0}\right)$ would be zero.

Finally, suppose that the extremal is abnormal and let $t_{0}$ be a switching point implying $\phi\left(t_{0}\right)=q\left(t_{0}\right)=0$. It follows that $p\left(t_{0}\right) \neq 0$ (otherwise the vector $\lambda$ would be zero on $\left[0, T_{u}\right]$ and this would contradict the PMP). Now, suppose that $y\left(t_{0}\right) \neq r$, then we find that $p\left(t_{0}\right) x\left(t_{0}\right)\left(r-y\left(t_{0}\right)\right) \neq 0$ which again contradicts the PMP as one has $p_{0}=0$. Hence, we necessarily have $y\left(t_{0}\right)=r$ which proves (iv).

Remark 4.1. From (4.2) and the fact that $\left(\lambda(\cdot), p_{0}\right)$ is non-zero, the mapping $t \longmapsto(p(t), q(t))$ is always non-zero. Using a similar argument as in the proof of the first point of Proposition 4.1, one can prove that the zeros of $\phi$ are isolated.

We now wish to synthesize an optimal feedback control for (4.1) using the previous analysis. We first analyze the behavior of the switching function $\phi$.

Lemma 4.2. A normal extremal trajectory $(z(\cdot), \lambda(\cdot), u)$ defined over $\left[0, T_{u}\right]$ satisfies the following properties: 
(i) The switching function is solution of the ordinary differential equation (ODE):

$$
\dot{\phi}(t)=\frac{y(t)(m+u(t)-x(t))}{r-y(t)} \phi(t)-\frac{y(t)}{r-y(t)}, \quad \text { a.e. } t \in\left[0, T_{u}\right],
$$

(ii) At a time $t_{0}$ where $y\left(t_{0}\right)=r$, we have $\phi\left(t_{0}\right) \neq 0$ and:

$$
\phi\left(t_{0}\right)=\frac{1}{u\left(t_{0}\right)+m-x\left(t_{0}\right)} .
$$

Proof. Let us first show that the set $S:=\left\{t \in\left[0, T_{u}\right] ; y(t)=r\right\}$ is finite. If $t_{0} \in S$, we have $q\left(t_{0}\right) y\left(t_{0}\right)\left(x\left(t_{0}\right)-\right.$ $\left.m-u\left(t_{0}\right)\right)=1$ which implies that $\dot{y}\left(t_{0}\right) \neq 0$, hence $t_{0}$ is isolated and thus $S$ is finite. Using that $\phi=-q y$, $\dot{\phi}=-p x y$, and that $H=0$, we get that (4.7) holds almost everywhere which proves (i). The proof of (ii) is straightforward combining $H=0$ and $y\left(t_{0}\right)=r$.

This Lemma leads to the following Proposition.

Proposition 4.2. Let $(z(\cdot), \lambda(\cdot), u)$ be a normal extremal trajectory defined over $\left[0, T_{u}\right]$. Then, the following properties hold true.

(i) If there exist two consecutive times $t_{2}>t_{1}>0$ such that $y\left(t_{1}\right)=y\left(t_{2}\right)=r$, then the control u has exactly one switching time $t_{c} \in\left(t_{1}, t_{2}\right)$.

(ii) If, in addition, one has $x\left(t_{1}\right)>x\left(t_{2}\right)$, resp. $x\left(t_{1}\right)<x\left(t_{2}\right)$, then an optimal control satisfies $u=0$, resp. $u=\bar{u}$ on $\left(t_{1}, t_{c}\right)$ and then $u=\bar{u}$, resp. $u=0$ on $\left(t_{c}, t_{2}\right)$.

Proof. From (4.8), the sign of $\phi\left(t_{i}\right), i=1,2$ depends on the value of $x\left(t_{i}\right)$ compared to $u\left(t_{0}\right)+m$. Whenever the trajectory satisfies $y\left(t_{1}\right)=r$ with $x\left(t_{1}\right)>x_{\bar{u}}^{+}(\underline{x})$, we thus have $\phi\left(t_{1}\right)<0$ implying $u=0$. Using the inequality $x\left(t_{2}\right)<m$, we deduce that $\phi\left(t_{2}\right)>0$, hence the trajectory necessarily has a switching point at some time $t_{c} \in\left(t_{1}, t_{2}\right)$. Now, from (4.7), one has $\dot{\phi}\left(t_{c}\right)=-\frac{y\left(t_{c}\right)}{r-y\left(t_{c}\right)}>0$. Thus, the only possibility for the trajectory is to switch from $u=0$ to $u=\bar{u}$. This shows the uniqueness of $t_{c}$ in $\left(t_{1}, t_{2}\right)$. If now $x\left(t_{1}\right)<x_{\bar{u}}^{+}(\underline{x})$, the same argumentation shows that there exists a unique switching time from $u=\bar{u}$ to $u=0$ in $\left(t_{1}, t_{2}\right)$. This ends the proof of the Proposition.

We denote by $\gamma$ the graph of the unique solution $(\tilde{x}(\cdot), \tilde{y}(\cdot))$ of $(3.1)$ backward in time starting from the point $(\underline{x}, r)$ associated to the feedback control (3.4). Let $\tau_{1}$ be the first time where $(\tilde{x}(\cdot), \tilde{y}(\cdot))$ exits $K(\underline{x})$ and $\tau_{2}>\tau_{1}$ be the first exit time of $(\tilde{x}(\cdot), \tilde{y}(\cdot))$ of the set $\{(x, y) \in \mathcal{D} ; y \leq r\}$. Finally, let $\gamma_{1}$ be the restriction of $(\tilde{x}(\cdot), \tilde{y}(\cdot))$ to the interval $\left[\tau_{1}, \tau_{2}\right]$. The optimal synthesis of the problem then reads as follows (see also Fig. 2).

Theorem 4.1. Let $\left(x_{0}, y_{0}\right)$ be an initial condition in $\mathcal{D} \backslash \operatorname{Viab}(\underline{x})$.

(i) If $\left(x_{0}, y_{0}\right) \in \gamma$, then any optimal trajectory of $(3.1)$ steering $\left(x_{0}, y_{0}\right)$ to the target set is abnormal. The corresponding control is given by $u_{m}(\cdot)$ and switching points occur on the line $\{y=r\}$.

(ii) If $\left(x_{0}, y_{0}\right) \notin \gamma$, then any optimal trajectory of (3.1) steering $\left(x_{0}, y_{0}\right)$ to the target set is normal. Moreover, if $u$ denotes the optimal control, there exists $p \in \mathbb{N}^{*}, s \in\{0,1\}$, and a sequence of times $\left(\tau_{k}\right)_{0 \leq k \leq p}$ such that:

- We have $\tau_{0}=0<\tau_{1}<\cdots<\tau_{p-1}<\tau_{p}=T_{u}$ and $\tau_{k}$ is a switching time of $u$ for $1 \leq k \leq p$.

- The optimal control $u$ is given by

$$
u(t)=\frac{\bar{u}}{2}\left(1+(-1)^{p-k-s}\right) \quad t \in\left(\tau_{k}, \tau_{k+1}\right), \quad 0 \leq k \leq p-1 .
$$

- If $y\left(T_{u}\right) \in\left(r_{-}, r\right)$, resp. $y\left(T_{u}\right)=r_{-}$, then $s=1$, resp. $s=0$. 
Proof. Let us prove (i) and take an initial condition on the curve $\gamma$. We already know from Proposition 4.1 that any trajectory starting on the curve $\gamma$ and associated to the control $u_{m}$ corresponds to an abnormal extremal trajectory. Indeed, such a trajectory remains on the curve $\gamma$, therefore switching points only occur on the axis $\{y=r\}$ implying that the trajectory is abnormal. We must prove that such an extremal is optimal. To do so, let us choose $\left(x_{0}, y_{0}\right)$ on the curve $\gamma$, and let $(x(\cdot), y(\cdot), \lambda(\cdot), u)$ be an optimal extremal trajectory steering $\left(x_{0}, y_{0}\right)$ to $\operatorname{Viab}(\underline{x})$. Let $t_{0}$ be defined as follows:

$$
t_{0}:=\inf \{t \geq 0 ; \exists \varepsilon>0 \forall \tau \in(t, t+\varepsilon) \quad(x(\tau), y(\tau)) \notin \gamma\} .
$$

Suppose by contradiction that $t_{0}<T_{u}$. As $(x(\cdot), y(\cdot), u)$ is extremal, (4.3) implies that $t_{0}$ is necessarily a switching time from $u=0$ to $u=\bar{u}$ or from $u=\bar{u}$ to $u=0$. We argue that $y\left(t_{0}\right) \neq r$. Indeed, otherwise, we would have a contradiction with the definition of $t_{0}$ (as by definition, $u_{m}$ switches on the line $\{y=r\}$ ). Hence, either we have $y\left(t_{0}\right)<r$ or $y\left(t_{0}\right)>r$. Now, the fact that $y\left(t_{0}\right) \neq r$ implies that the extremal trajectory is a normal one. Indeed, we cannot have $p\left(t_{0}\right)=0$ by Cauchy-Lipschitz's Theorem, but as $y\left(t_{0}\right) \neq r$, we obtain that $p\left(t_{0}\right) x\left(t_{0}\right)\left(r-y\left(t_{0}\right)\right) \neq 0$ and thus $p_{0}$ must be non null. Suppose for instance that $y\left(t_{0}\right)<r$. By construction of $t_{0}$, this point is a switching time from $u=0$ to $u=\bar{u}$ and we necessarily have $\dot{\phi}\left(t_{0}\right) \geq 0$. On the other hand, we obtain from (4.7) that

$$
\dot{\phi}\left(t_{0}\right)=-\frac{y\left(t_{0}\right)}{r-y\left(t_{0}\right)}<0
$$

and thus a contradiction. If $y\left(t_{0}\right)>r$, we obtain a similar contradiction with the sign of $\dot{\phi}$ at time $t_{0}$. This shows that $t_{0} \geq T_{u}$, thus we have proved that the abnormal extremal trajectory starting from $\left(x_{0}, y_{0}\right)$ with the control $u_{m}(\cdot)$ drives a solution of (3.1) optimally to the target.

Let us prove (ii). The first two properties follow from Proposition 4.1 and from the fact that the number of switching times of an optimal control is finite. Given an extremal trajectory $(x(\cdot), y(\cdot), \lambda(\cdot), u)$ driving optimally $\left(x_{0}, y_{0}\right) \notin \gamma$ to $\operatorname{Viab}(\underline{x})$, we consider two cases depending if $y\left(T_{u}\right) \in\left(r_{-}, r\right)$ or $y\left(T_{u}\right)=r_{-}$.

First case: $y\left(T_{u}\right) \in\left(r_{-}, r\right)$. From Proposition 4.1, we have $p_{0} \neq 0$ i.e. the trajectory is normal. Now, as $\phi\left(T_{u}\right)=0$ and $\dot{\phi}\left(T_{u}\right)=-\frac{y\left(T_{u}\right)}{r-y\left(T_{u}\right)}<0$, we obtain that $u=\bar{u}$ in a left neighborhood of $T_{u}$. By using Proposition 4.2 , we obtain that the extremal has exactly one switching time $t_{c}$ between two consecutive instants $t_{1}<t_{2}$ such that $y\left(t_{1}\right)=y\left(t_{2}\right)=r$. We thus obtain (4.9) by considering (3.1) backward in time from $t=T_{u}$ and by counting the number of times (denoted by $p-1$ with $p \geq 1$ ) where the trajectory surrounds $V i a b(\underline{x})$ before reaching $\left(x_{0}, y_{0}\right)$. When $k=p-1$, we obtain $u(t)=\frac{\bar{u}}{2}\left(1+(-1)^{1-s}\right)$, thus $s=1$ as was to be proved.

Second case: $y\left(T_{u}\right)=r_{-}$. Suppose that the extremal is abnormal i.e. $p_{0}=0$. It follows that $q\left(T_{u}\right)>0$. Otherwise, the transversality condition would imply $q\left(T_{u}\right)=0$ and using $H=0$ we would have $p\left(T_{u}\right)=0$ and a contradiction with the PMP. We deduce that $\phi\left(T_{u}\right)<0$ thus $u=0$ in a left neighborhood of $T_{u}$. As the extremal is abnormal, switching points occur only on the line $\{y=r\}$. This shows that we have $u=0$ on $\left[t_{c}, T_{u}\right]$ where $t_{c}$ is the last time such that $y\left(t_{c}\right)=r$ before reaching $B^{0}(\underline{x})$. Thus, by integrating backward in time (3.1) from $\left(\underline{x}, r_{-}\right)$, we find that $u=u_{m}(\cdot)$ and that $\left(x_{0}, y_{0}\right) \in \gamma$ which is a contradiction. We have thus proved that the extremal optimal trajectory is normal. Finally, we have two cases depending on whether the optimal trajectory reaches $\left(\underline{x}, r_{-}\right)$with either the control $u=0$ or $u=\bar{u}$ :

- The case where we have $u=\bar{u}$ at the terminal time $T_{u}$ is similar to the first case $y\left(T_{u}\right) \in\left(r_{-}, r\right)$ above. Thus, the conclusion is obtained similarly as above.

- Now, suppose that we have $u=0$ at the terminal time $T_{u}$. The trajectory necessary has a switching time on $\gamma_{1}$ (as it is normal). We thus obtain (4.9) by considering (3.1) backward in time from $t=T_{u}$ and by counting the number of times (denoted by $p-1$ with $p \geq 1$ ) where the trajectory surrounds $\operatorname{Viab}(\underline{x})$ before reaching $\left(x_{0}, y_{0}\right)$. As $u=0$ in a left neighborhood of $T_{u}$, we obtain $s=0$.

When the viability kernel is reduced to the singleton $\{(\underline{x}, r)\}$ i.e. when $\bar{u}=\underline{x}-m$, Theorem 3.1 still holds true, even though there is no transversality condition on the terminal adjoint vector. This can be interpreted as the limiting case when the two extreme points of $B^{0}(\underline{x})$ collapse. 


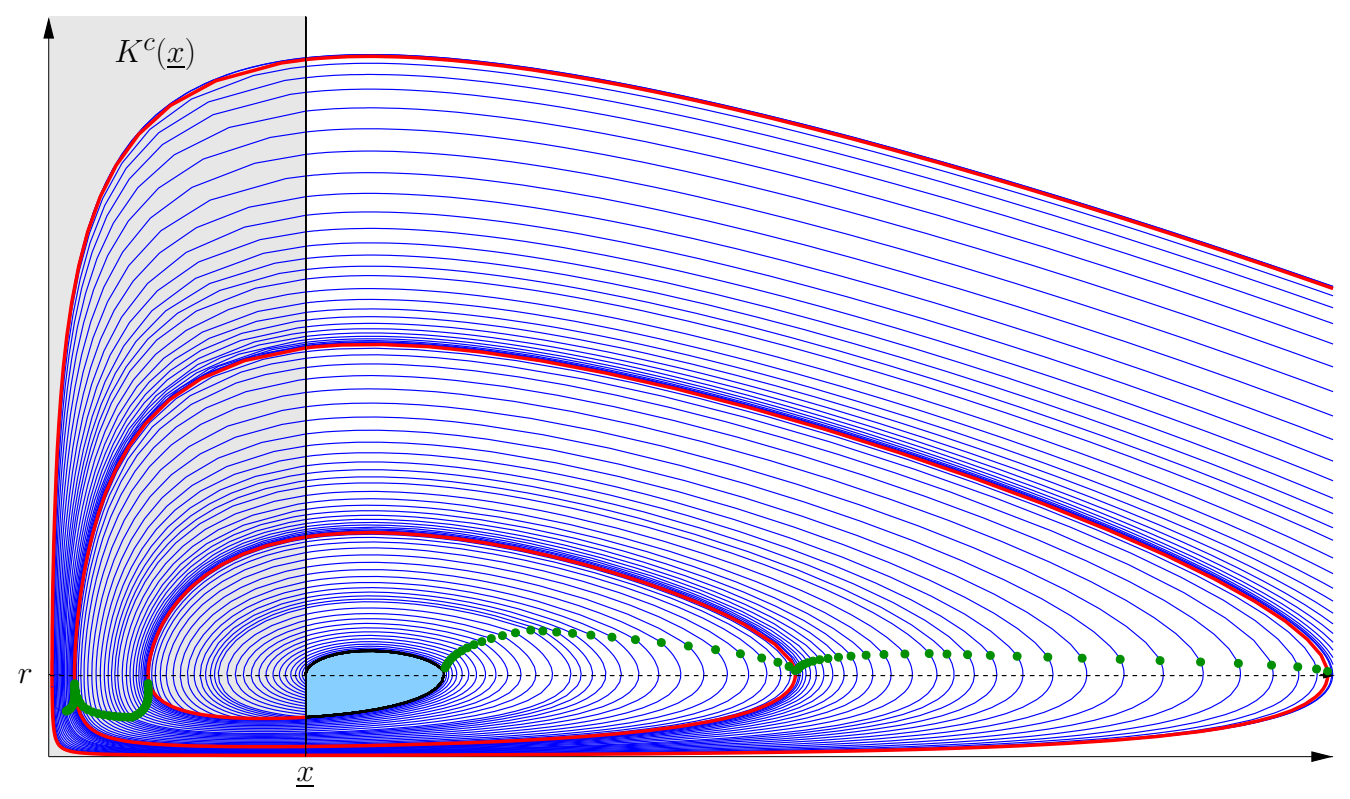

Figure 2: Examples of optimal trajectories for the minimal time to reach $V i a b(\underline{x})$ (see Appendix for the numerical values). In blue, normal optimal trajectories reaching the target set at $B_{0}(\underline{x})$. In red, the abnormal optimal trajectory reaching $B_{0}(\underline{x})$ at the extreme point $\left(\underline{x}, r_{-}\right)$. Switches (from 0 to $\bar{u}$ or from $\bar{u}$ to 0 ) are represented by the green dots.

\section{Minimal time crisis versus minimal time}

In this section, our aim is twofold. We first compute the optimal synthesis for the minimal time crisis problem. We then compare compare the time spent in $K^{c}$ for the two strategies (minimal time to reach $V i a b(\underline{x})$ and minimal time crisis).

\subsection{Optimal synthesis for the minimal time crisis problem}

According to (3.3), the minimal time crisis problem can be stated as

$$
\inf _{\tau \geq 0, u \in \mathcal{U}} \int_{0}^{\tau} \mathbb{1}_{K^{c}}\left(z\left(t, z_{0}\right)\right) \mathrm{d} t \quad \text { s.t. } z\left(\tau, z_{0}\right) \in \operatorname{Viab}(\underline{x}) .
$$

Let $H: \mathbb{R}^{2} \times \mathbb{R}^{2} \times \mathbb{R} \times \mathbb{R} \rightarrow \mathbb{R}$ be the Hamiltonian associated to (5.1) defined by:

$$
H:=H\left(x, y, p, q, p_{0}, u\right)=p x(r-y)+q y(x-m-u)+p_{0} \mathbb{1}_{K(\underline{x})^{c}}(x, y) .
$$

If $u$ is an optimal control of (5.1) defined over a time interval $[0, \tau]$, and $(x, y)$ is the associated solution of (3.1), then the following optimality conditions are satisfied:

- There exist numbers $\tau \geq 0, p_{0} \leq 0$ and a measurable function $\lambda(\cdot):=(p(\cdot), q(\cdot)):[0, \tau] \rightarrow \mathbb{R}^{2}$ that is almost everywhere absolutely continuous, satisfying a.e. on $[0, \tau]$ :

$$
\left\{\begin{array}{l}
\dot{p}=p(y-r)-q y, \\
\dot{q}=p x+q(u+m-x) .
\end{array}\right.
$$

- The control $u$ satisfies the maximization condition:

$$
u(t) \in \arg \max _{\omega \in[0, \bar{u}]} H\left(x(t), y(t), p(t), q(t), p_{0}, \omega\right) \quad \text { a.e. } t \in[0, \tau] .
$$

- The Hamiltonian $H$ is constant equal to zero along any extremal trajectory $\left(x(\cdot), y(\cdot), p(\cdot), q(\cdot), p_{0}, u\right)$ satisfying (3.1)-(5.2)-(5.3) (we recall that the terminal time is free). 
- The covector is discontinuous at any crossing time $t_{c}\left(\right.$ from $K(\underline{x})$ to $K(\underline{x})^{c}$ or from $K(\underline{x})^{c}$ to $\left.K(\underline{x})\right)$ :

$$
\lambda\left(t_{c}^{+}\right)-\lambda\left(t_{c}^{-}\right) \in N_{K(\underline{x})}\left(x\left(t_{c}\right), y\left(t_{c}\right)\right) .
$$

- The triplet $\left(p_{0}, p(\cdot), q(\cdot)\right)$ is non identically null.

- The transversality condition $\lambda(T) \in-N_{\operatorname{Viab}(\underline{x})}(x(T), y(T))$ is equivalent to (4.6).

As for the minimal time control problem, one introduces the switching function $\phi:=-q y$ which satisfies:

$$
\left\{\begin{array}{l}
\phi(t)>0 \quad \Rightarrow \quad u(t)=\bar{u}, \\
\phi(t)<0 \quad \Rightarrow \quad u(t)=0, \\
\phi(t)=0 \quad \Rightarrow \quad u(t) \in[0, \bar{u}] .
\end{array}\right.
$$

Moreover, the adjoint equation implies $\dot{\phi}=-p x y$. Following Definition 2.3 for the set $K(\underline{x})$, a crossing time $t_{c}$ is transverse when one has $\dot{z}\left(t_{c}, z_{0}\right) . e_{1} \neq 0$, where $e_{1}$ denotes the vector $(1,0)$. We begin by two Lemmas that characterize crossing times.

Lemma 5.1. Given a solution $z\left(\cdot, z_{0}\right)$ of $(3.1)$, any crossing time $t_{c}$ of $z\left(\cdot, z_{0}\right)$ such that $z\left(t_{c}, z_{0}\right) \notin V i a b(\underline{x})$ is transverse.

Proof. Suppose that a solution $z\left(\cdot, z_{0}\right)=(x(\cdot), y(\cdot))$ of $(3.1)$ hits tangentially the boundary of $K(\underline{x})$ at some point $(\underline{x}, y)$. Then, we must have $\dot{z}\left(t_{c}, z_{0}\right) \cdot e_{1}=0$ which implies that $y\left(t_{c}\right)=r$. Hence, we obtain that $\left(x\left(t_{c}\right), y\left(t_{c}\right)\right)=(\underline{x}, r) \in \operatorname{Viab}(\underline{x})$ which contradicts the hypothesis of the Lemma. Hence, any crossing time is transverse.

Thanks to this Lemma, we can write the jump condition on the adjoint vector as follows (see [4]). Let $t_{c}$ be a crossing time. Then, one has:

$$
\left\{\begin{array}{l}
x\left(t_{c}^{-}\right)>\underline{x} \text { and } x\left(t_{c}^{+}\right)<\underline{x} \quad \Rightarrow \quad p\left(t_{c}^{+}\right)-p\left(t_{c}^{-}\right)=\frac{q\left(t_{c}\right) y\left(t_{c}\right)\left(u\left(t_{c}^{+}\right)-u\left(t_{c}^{-}\right)\right)-p_{0}}{\underline{x}\left(r-y\left(t_{c}\right)\right)} \\
x\left(t_{c}^{-}\right)<\underline{x} \text { and } x\left(t_{c}^{+}\right)>\underline{x} \quad \Rightarrow \quad p\left(t_{c}^{+}\right)-p\left(t_{c}^{-}\right)=\frac{q\left(t_{c}\right) y\left(t_{c}\right)\left(u\left(t_{c}^{+}\right)-u\left(t_{c}^{-}\right)\right)+p_{0}}{\underline{x}\left(r-y\left(t_{c}\right)\right)}
\end{array}\right.
$$

and the function $q(\cdot)$ is (absolutely) continuous over $[0, T]$ whereas $p$ is piece-wise (absolutely) continuous. We then obtain the following characterization of the jumps.

Lemma 5.2. Let us consider an extremal trajectory $\left(x(\cdot), y(\cdot), p(\cdot), q(\cdot), p_{0}, u\right)$.

(i) If the extremal is abnormal (i.e. $\left.p_{0}=0\right)$, then the adjoint vector $(p(\cdot), q(\cdot))$ is absolutely continuous.

(ii) If the extremal is normal (i.e. $p_{0}<0$ ), then a crossing time $t_{c}$ is such that

$$
\left\{\begin{array}{l}
x\left(t_{c}^{-}\right)>\underline{x} \text { and } x\left(t_{c}^{+}\right)<\underline{x} \quad \Rightarrow \quad p\left(t_{c}^{+}\right)-p\left(t_{c}^{-}\right)=\frac{1}{\underline{x}\left(r-y\left(t_{c}\right)\right)} \\
x\left(t_{c}^{-}\right)<\underline{x} \text { and } x\left(t_{c}^{+}\right)>\underline{x} \quad \Rightarrow \quad p\left(t_{c}^{+}\right)-p\left(t_{c}^{-}\right)=\frac{-1}{\underline{x}\left(r-y\left(t_{c}\right)\right)} .
\end{array}\right.
$$

Proof. Let us first show that $q\left(t_{c}\right) y\left(t_{c}\right)\left(u\left(t_{c}^{+}\right)-u\left(t_{c}^{-}\right)\right)$is zero at any crossing time. The result is obvious if $q\left(t_{c}\right)=0$. Now, if $q\left(t_{c}\right)<0$, then $\phi>0$ in a neighborhood of $t_{c}$, thus $u=\bar{u}$ in a neighborhood of $t_{c}$ (recall that $\phi$ is continuous) so that $u\left(t_{c}^{+}\right)-u\left(t_{c}^{-}\right)=0$. The same conclusion follows if $q\left(t_{c}\right)>0$. Using the equality $q\left(t_{c}\right) y\left(t_{c}\right)\left(u\left(t_{c}^{+}\right)-u\left(t_{c}^{-}\right)\right)=0$, one obtains straightforwardly (i) and (ii) from (5.5).

We can now state our main result that characterize the optimal solutions of Problem (5.1).

Proposition 5.1. Consider an optimal solution of Problem (5.1) defined over $[0, T]$.

(1) If $\left(x_{0}, y_{0}\right) \in \gamma$, then the optimal trajectory is abnormal and the optimal control is given by $u_{m}$. Switching points occur on the line $\{y=r\}$. 
(2) If $\left(x_{0}, y_{0}\right) \notin \gamma$, then the optimal trajectory is normal. Moreover, the following properties hold true:

(i) If $\tau$ is the last instant for which $y(\tau)=r$, then one has $u=\bar{u}$ over $[\tau, T]$.

(ii) Any switching point in $K(\underline{x})^{c} \cap\{y>r\}$ is from $u=0$ to $u=\bar{u}$.

(iii) Any switching point in $K(\underline{x})^{c} \cap\{y<r\}$ is from $u=\bar{u}$ to $u=0$.

(iv) If a switching point occurs in $K(\underline{x})$ at an instant $t_{s}$, then we must have $y\left(t_{s}\right)=r$.

Proof. First, notice that $\mathbb{1}_{K(\underline{x})^{c}}$ is zero in $K(\underline{x})$, hence any switching time $t_{s}$ that occurs in the set $K(\underline{x})$ necessarily satisfies $y\left(t_{s}\right)=r$.

To prove (1), we suppose by contradiction (as in the proof of the first point in Theorem 4.1) that the trajectory starting from $\left(x_{0}, y_{0}\right) \in \gamma$ contains a switching point $t_{s}$ such that $q\left(t_{s}\right)=0$ and $y\left(t_{s}\right) \neq r$. We may suppose that $t_{s}$ is the first one satisfying $y\left(t_{s}\right) \neq r$. Hence, the trajectory is normal (otherwise, the condition $H=0, q\left(t_{s}\right)=0$ and $y\left(t_{s}\right) \neq r$ would imply a contradiction). Finally, suppose that $y\left(t_{s}\right)>r$. Thus, $t_{s}$ is a switching point from $u=\bar{u}$ to $u=0$, i.e. $\phi\left(t_{s}\right)=0$ implying also $\dot{\phi}\left(t_{s}\right) \leq 0$. From (4.7) (which remains valid in $K(\underline{x})^{c}$ ) we deduce that $\dot{\phi}\left(t_{s}\right)<0$ which is a contradiction. If now $y\left(t_{s}\right)<r$, then $t_{s}$ is by construction a switching point from $u=0$ to $u=\bar{u}$, and we obtain a similar contradiction. Hence, we deduce that the optimal control is $u_{m}$ and that the corresponding trajectory is abnormal.

To prove (2), we use the transversality condition (4.6) which implies that either $p(T)>0$ and $q(T)=0$, thus $\dot{\phi}\left(T^{-}\right) \leq 0$ (when $y(t) \in\left(r_{-}, r\right)$ ) or $q(T)<0$ (when $y(t)=r_{-}$). Suppose that the trajectory reaches $B^{0}(\underline{x})$ in its interior. Then, one must have $p(T) \underline{x}(r-y(T))+p_{0}=0$, hence $p_{0}<0$ and the trajectory is normal (otherwise we would have $p(\cdot)$ and $q(\cdot)$ identically equal to zero which contradicts the hybrid maximum principle). Suppose now that the trajectory reaches the point $\left(\underline{x}, r_{-}\right)$at time $t=T$. Then, if the trajectory is abnormal, it must coincide with the curve $\gamma$ (as switching points only occur on the line $\{y=r\}$. Thus we obtain a contradiction with $\left(x_{0}, y_{0}\right) \notin \gamma$.

It follows that one has $\phi>0$ in a left neighborhood of $T$ and thus there exists $\tau>0$ such that the control satisfies $u=\bar{u}$ over $[\tau, T]$ which gives (i). Now, thanks to (4.7), we obtain that any switching point in $K(\underline{x})^{c} \cap\{y>r\}$, resp. $K(\underline{x})^{c} \cap\{y<r\}$ is from $u=0$ to $u=\bar{u}$, resp. from $u=\bar{u}$ to $u=0$ implying (ii)-(iii). Finally, note that in the set $K(\underline{x})$, the Hamiltonian writes

$$
H=p x(r-y)+q y(x-m-u)=0,
$$

implying that $y\left(t_{s}\right)=r$ whenever $q\left(t_{s}\right)=0$. This ends the proof.

We then deduce the following result.

Corollary 5.1. Let $(x(\cdot), y(\cdot))$ be a normal extremal trajectory defined over a time interval $\left[t_{0}, t_{2}\right]$ such that :

- At time $t_{0}$, one has $y\left(t_{0}\right)=r,\left(x\left(t_{0}\right), y\left(t_{0}\right)\right) \in K(\underline{x}) \backslash V i a b(\underline{x})$, and $t_{0}$ is a switching point from $u=0$ to $u=\bar{u}$.

- There exists $t_{1} \in\left(t_{0}, t_{2}\right)$ such that $x\left(t_{1}\right)=x\left(t_{2}\right)=\underline{x}$ with $\left(x\left(t_{2}\right), y\left(t_{2}\right)\right) \notin V i a b(\underline{x})$ and $t_{1}<t_{2}$ are two consecutive crossing times from $K(\underline{x})$ to $K(\underline{x})^{c}$ and from $K(\underline{x})^{c}$ to $K(\underline{x})$ respectively.

Then, the trajectory has exactly one switching time $t_{s} \in\left(t_{1}, t_{2}\right)$ from $u=\bar{u}$ to $u=0$ such that $y\left(t_{s}\right)<r$.

Proof. As $t_{0}$ is a switching point such that $\left(x\left(t_{0}\right), y\left(t_{0}\right)\right) \in K(\underline{x}) \backslash V i a b(\underline{x})$, we necessarily have $u=\bar{u}$ over $\left[t_{0}, t_{1}\right)$. Now, using that $q(\cdot)$ is continuous and that no switching points occur in the interval $\left(t_{0}, t_{1}\right]$, we must have $q\left(t_{1}\right)<0$ thus $\phi\left(t_{1}\right)>0$, and consequently one has $\phi>0$ in a right neighborhood of $t_{1}$. From Proposition 5.1 , the trajectory cannot switch from $u=\bar{u}$ to $u=0$ in the set $K(\underline{x})^{c} \cap\{y>r\}$. Recall that one has $\phi>0$ when the trajectory crosses the line $\{y=r\}$. Suppose now that the trajectory does not switch in the set $K(\underline{x})^{c} \cap\{y<r\}$. Then, one has $\phi>0$ until $t=t_{2}$. At this time, the trajectory enters $K(\underline{x})$ with $\phi>0$ (as $q$ is continuous), thus we have $u=\bar{u}$ until that the trajectory again reaches the point $\left(x\left(t_{0}\right), r\right)$. Indeed, recall that switching points in $K(\underline{x})$ only occur on the axis $\{y=r\}$. This contradicts the optimality of the trajectory. Hence, there must exist a switching point in the set $K(\underline{x})^{c} \cap\{y<r\}$ as was to be proved.

Typical optimal trajectories are depicted on Fig. 3 (see Appendix for details on the numerical simulations). Switching points are represented in black. Switching curves consist of the collection of these points. 


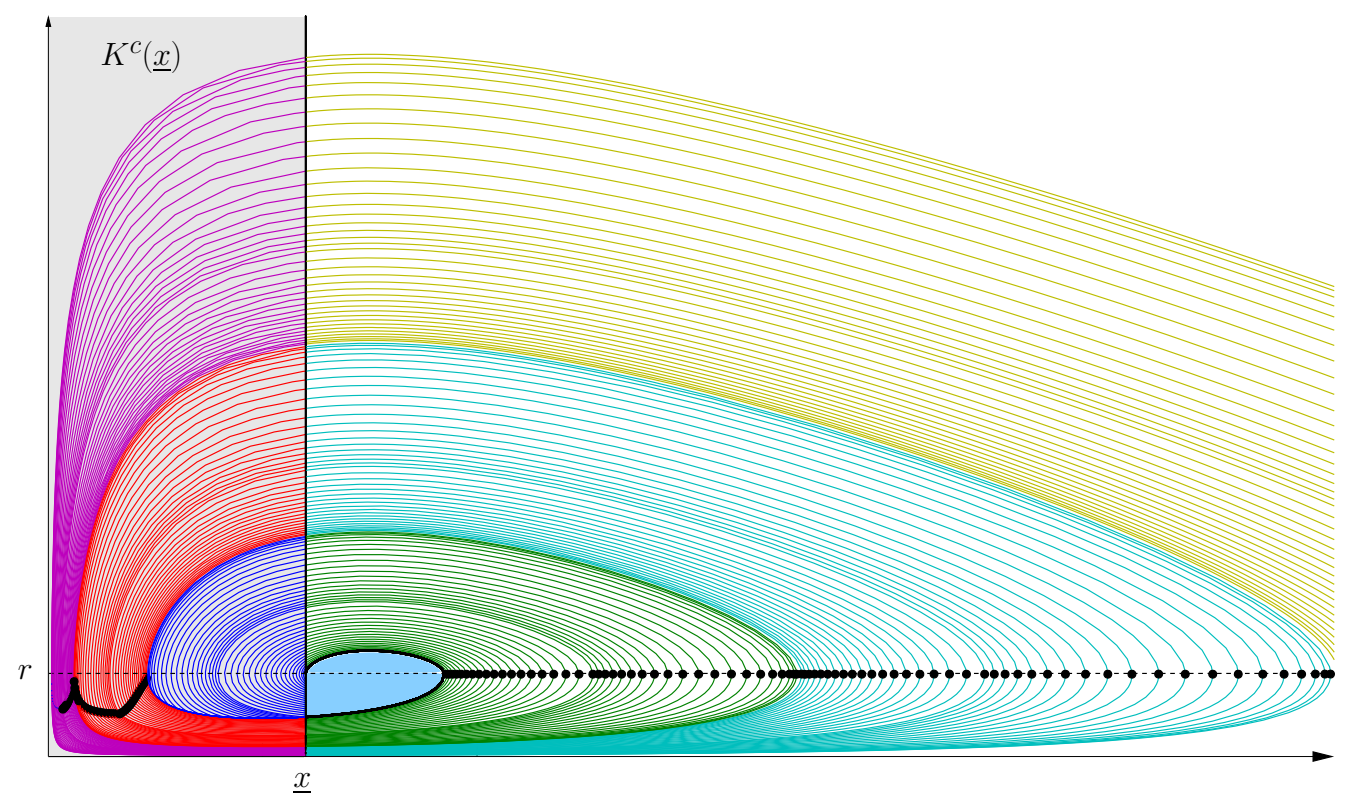

Figure 3: Examples of normal optimal trajectories for the minimal time crisis (see Appendix for the numerical values). Color of the trajectories are changed at each crossing time. Switching points are represented by the black dots.

\subsection{Comparison with the minimal time strategy}

In order to compare solutions of Problems (4.1) and (5.1), we consider the subset $\mathcal{E} \subset \mathcal{D}$ containing all the points of $\mathcal{D}$ that can reach $\operatorname{Viab}(\underline{x})$ with the constant control $u=\bar{u}$ and such that the corresponding optimal trajectory does not contain any switching point. Moreover, let $\mathcal{F}$ be defined by:

$$
\mathcal{F}:=\mathcal{E} \cup \gamma .
$$

Proposition 5.2. Given an initial condition $z_{0}=\left(x_{0}, y_{0}\right) \in \mathcal{D}$, we have the two following cases:

(i) If $z_{0} \in \mathcal{F}$, optimal solutions for Problems (4.1) and (5.1) coincide.

(ii) If $z_{0} \in \mathcal{D} \backslash \mathcal{F}$, then one has

$$
\theta\left(z_{0}\right)<J\left(u^{\star}, z_{0}\right),
$$

where $u^{\star}$ denotes an optimal control for (4.1).

Proof. The proof of (i) is immediate from Theorem 4.1 and Proposition 5.1. Take now an initial condition $z_{0}=\left(x_{0}, y_{0}\right) \in \mathcal{D} \backslash \mathcal{F}$ and let $u^{\star}$ be an optimal control for (4.1). As $u^{\star} \in \mathcal{U}$ is admissible for (5.1), we have $\theta\left(z_{0}\right) \leq J\left(u^{\star}, z_{0}\right)$. If we have $\theta\left(z_{0}\right)=J\left(u^{\star}, z_{0}\right)$, then $u^{\star}$ is necessarily an optimal control for (5.1). The switching points in $K(\underline{x})$ of the associated trajectory occur on the line $\{y=r\}$ only from Proposition 5.1. As $z_{0} \in \mathcal{D} \backslash \mathcal{F}$ the trajectory necessarily has at least one switching point in the set $K(\underline{x})$ from Proposition 4.2 at some time $t_{c}$. At time $t_{c}$, we must have $y\left(t_{c}\right)>r$ as the corresponding trajectory is a normal extremal (see Theorem 4.1). This gives a contradiction and proves (ii).

Intuitively, this result says that for initial conditions in $\mathcal{F}$, the time spent in $K^{c}$ by an optimal trajectory of the minimal time problem (4.1) is greater than the one spent in in $K^{c}$ by an optimal trajectory of the time crisis problem (5.1). To conclude this study, we provide properties of the minimal time crisis problem when the viability kernel $\operatorname{Viab}(\underline{x})$ is empty, that is when the condition $m+\bar{u}<\underline{x}$ is fulfilled (see Proposition 3.1).

Proposition 5.3. When $m+\bar{u}<\underline{x}$, the following properties hold true.

(i) There is no chattering phenomenon for Problem 5.1 in the sense of Definition 2.2 for system (3.1). 
(ii) For any $z_{0} \in \mathcal{D}$, one has $\theta\left(z_{0}\right)=+\infty$.

Proof. First, suppose that there exist two sequences of times $\left(t_{n}^{1}\right)$ and $\left(t_{n}^{2}\right)$ satisfying:

- both sequences $\left(t_{n}^{1}\right)$ and $\left(t_{n}^{2}\right)$ are increasing with $t_{n}^{1}<t_{n}^{2}$ for any $n \in \mathbb{N}$ and such that $t_{n}^{2}-t_{n}^{1} \rightarrow 0$ when $n \rightarrow+\infty$.

- for any $n \in \mathbb{N}, t_{n}^{1}$, resp. $t_{n}^{2}$ is a crossing time from $K(\underline{x})$ to $K(\underline{x})^{c}$, resp. from $K(\underline{x})^{c}$ to $K(\underline{x})$,

- for any time $t \in\left(t_{n}^{1}, t_{n}^{2}\right)$, one has $(x(t), y(t)) \in K(\underline{x})^{c}$.

As $m+\bar{u}<\underline{x}$, there exists $\varepsilon>0$ such that $m+\bar{u}+\varepsilon<\underline{x}$. Let us now integrate (3.1) over $\left[t_{n}^{1}, t_{n}^{2}\right]$. Since $u(t) \leq \bar{u}$ for any time $t$, one has:

$$
\int_{y_{n}^{1}}^{y_{n}^{2}} \frac{\mathrm{d} y}{y}=\int_{t_{n}^{1}}^{t_{n}^{2}}(x(t)-m-u(t)) \mathrm{d} t \geq \int_{t_{n}^{1}}^{t_{n}^{2}}(x(t)-m-\bar{u}) \mathrm{d} t>\int_{t_{n}^{1}}^{t_{n}^{2}}(x(t)-\underline{x}+\varepsilon) \mathrm{d} t,
$$

where $y_{n}^{1}:=y\left(t_{n}^{1}\right)$ and $y_{n}^{2}:=y\left(t_{n}^{2}\right)$, or equivalently

$$
\varepsilon\left(t_{n}^{2}-t_{n}^{1}\right)+\ln \left(\frac{y_{n}^{1}}{y_{n}^{2}}\right)<\int_{t_{n}^{1}}^{t_{n}^{2}}(\underline{x}-x(t)) \mathrm{d} t .
$$

As one should have at the crossing times $\dot{x}\left(t_{n}^{1}\right)>0$ and $\dot{x}\left(t_{n}^{2}\right)<0$, one immediately obtains from equations (3.1) the inequalities $y_{n}^{1}>r>y_{n}^{2}$, and thus $\ln \left(\frac{y_{n}^{1}}{y_{n}^{2}}\right)$ is a positive number, for any $n$. We then deduce from (5.7) that $\ln \left(\frac{y_{n}^{1}}{y_{n}^{2}}\right)$ has to tend to 0 when $n$ tends to $+\infty$, which implies that $y_{n}^{1}$ and $y_{n}^{2}$ both tend to $r$. Therefore, $y(\cdot)$ is uniformly bounded on the intervals $\left[t_{n}^{1}, t_{n}^{2}\right]$, say by a number $C>0$. It follows that one has

$$
\forall n \in \mathbb{N}, \forall t \in\left[t_{n}^{1}, t_{n}^{2}\right], \quad|\dot{x}(t)| \leq x(t)(r+y(t)) \leq A,
$$

where $A:=\underline{x}(r+C)$. We then deduce that $|x(t)-\underline{x}| \leq A\left|t-t_{n}^{1}\right|$ for any $n \in \mathbb{N}$ and any time $t \in\left[t_{n}^{1}, t_{n}^{2}\right]$. Finally one has from $(5.7)$

$$
\varepsilon\left(t_{n}^{2}-t_{n}^{1}\right)+\ln \left(\frac{y_{n}^{1}}{y_{n}^{2}}\right)<\frac{A}{2}\left(t_{n}^{2}-t_{n}^{1}\right)^{2}
$$

which gives a contradiction for large values of $n$. This concludes the proof (i).

To prove (ii), suppose by contradiction that there exists $z_{0} \in \mathcal{D}$ such that $\theta\left(z_{0}\right)<+\infty$ and let $(x(\cdot), y(\cdot), u)$ be an optimal solution. Then, $x(\cdot)$ has an infinite number of crossing times. Otherwise, either $x(\cdot)$ remains in $K(\underline{x})$ after a certain time $\tau \geq 0$, which is a contradiction with $\operatorname{Viab}(\underline{x})=\emptyset$, or it remains in $K(\underline{x})^{c}$ after a certain time $\tau \geq 0$, which is a contradiction with $\theta\left(z_{0}\right)<+\infty$. Without any loss of generality, we can then suppose that there exist two sequences of times $\left(t_{n}^{1}\right),\left(t_{n}^{2}\right)$ as above. We then obtain a contradiction as previously, which ends the proof.

Remark 5.1. (i) This result shows that even if $\theta\left(z_{0}\right)=+\infty$, then no chattering phenomenon occurs.

(ii) Proposition 5.3 implies that when $m+\bar{u}<\underline{x}$ one has $J\left(u, z_{0}\right)=+\infty$ for any $u \in \mathcal{U}$ and $z_{0} \in \mathcal{D}$. However, it is possible to study the minimal time crisis problem restricted to a given finite horizon (see [4, 5]) and to characterize optimal controls in the same way.

\section{Conclusion and perspectives}

In this work, we first provided a reformulation of the minimal time crisis problem in general setup as a free terminal control problem. Thanks to this result, we could state optimality conditions using the hybrid principle provided that optimal trajectories cross the constraint set transversally. We have then applied this result to compute the minimal time crisis for the Lotka-Volterra prey-predator model when the control acts as a mortality term on the predators. This was made possible after an exact determination of the viability kernel. Finally, we have depicted a subset of the state space which is as follows: for any initial condition in this set, the time spent in the crisis set by optimal trajectories of the minimal time crisis problem is strictly less than 
the one spent by optimal trajectories of the minimal time problem reaching the viability kernel. Therefore, the minimal time crisis function appears to be an interesting alternative to the strategy which consists in steering a system in minimal time to the viability kernel. The methodology we have deployed here could be applied to other Lotka-Volterra equations or more general prey-predator models in order to highlight similar phenomena. As well, further works could focus on the study of the long-run averaged minimal time crisis problem in the case where the viability kernel is empty.

\section{Acknowledgments}

The authors thank the FMJH Gaspard Monge Program in Optimization and Operation Research, the ECOSSUD project C13E03, and the MathAm-SUD project SOCDE. The first author would like to thank INRA Montpellier and the UMR MISTEA for providing him a half-year delegation during the 2017-2018 academic year. The authors are grateful to Camila Romero for helpful discussions on the subject.

\section{Appendix: numerical simulations}

Numerical simulations for obtaining Fig. 2 and 3, have been conducted with the parameters $r=m=1$, $\bar{u}=0.5$ and $\bar{x}=1.2$. We proceed as follows. Given a terminal time $T>0$, we consider the state-adjoint system backward in time:

$$
\left\{\begin{aligned}
\dot{x} & =-x(r-y), \\
\dot{y} & =-y(x-m-u), \\
\dot{p} & =-p(y-r)+q y, \\
\dot{q} & =-p x-q(u+m-x),
\end{aligned}\right.
$$

over $[0, T]$ together with the control law $u(t)=\operatorname{sign}(-q(t))$ for a.e. $t \in[0, T]$ obtained from (5.3). The initialization makes use of the transversality condition (4.6) for an initial condition $\left(x_{0}, r\right)$ with $x_{0} \in\left[r_{-}, r\right)$.

Numerical determination of extremal trajectories for the minimum time problem (4.1).

First case. If $x_{0} \in\left(r_{-}, r\right)$, then $q(0)=0$ and $p(0)=\frac{1}{\underline{x}\left(r-x_{0}\right)}$ (thanks to $\left.H=0\right)$. Thus, (8.1) is initialized by the quadruple:

$$
\left(\underline{x}, x_{0}, \frac{1}{\underline{x}\left(r-x_{0}\right)}, 0\right) .
$$

Second case. If $x_{0}=r_{-}$, then there exists $\alpha \geq 0$ and $\beta \in[0,1]$ such that

$$
(p(0), q(0))=\alpha\left(1-\beta\left(1+w_{1}\right),-\beta w_{2}\right) \quad \text { and } \quad \alpha=\frac{1}{\underline{x}\left(r-r_{-}\right)\left(1-\beta\left(1+w_{1}\right)\right)-\beta w_{2} r_{-}(\underline{x}-m)},
$$

using the fact that the Hamiltonian is zero along any extremal trajectory. The system (8.1) is then initialized by the quadruple:

$$
\left(\underline{x}, r_{-}, p(0), q(0)\right) \text {, }
$$

with $(p(0), q(0))$ and $\alpha$ given by (8.3). Notice that in this case, the value of $\beta \in[0,1]$ is a parameter $($ as $K(\underline{x})$ is non-smooth at $\left(\underline{x}, r_{-}\right)$, there exist infinitely many extremal trajectories arising from $\left.\left(\underline{x}, r_{-}\right)\right)$.

Numerical determination of extremal trajectories for the minimum time crisis problem (5.1). The initialization of (8.1) is the same as for Problem (4.1). Moreover, the equation (8.1) remains valid as long as the trajectory does not belong to the boundary of $K(\underline{x})$. We thus impose the following condition:

$$
\left\{\begin{array}{l}
x\left(t_{c}^{-}\right)<\underline{x} \text { and } x\left(t_{c}^{+}\right)>\underline{x} \Rightarrow p\left(t_{c}^{+}\right)-p\left(t_{c}^{-}\right)=\frac{1}{\underline{x}\left(r-y\left(t_{c}\right)\right)} \\
x\left(t_{c}^{-}\right)>\underline{x} \text { and } x\left(t_{c}^{+}\right)<\underline{x} \Rightarrow p\left(t_{c}^{+}\right)-p\left(t_{c}^{-}\right)=\frac{-1}{\underline{x}\left(r-y\left(t_{c}\right)\right)}
\end{array}\right.
$$

at each crossing time $t_{c}$ (recall that according to the Hybrid Maximum Principle applied on Problem (5.3) only $p$ is discontinuous). Finally, the plots of optimal trajectories for (4.1) and (5.1) have been obtained by the scheme:

- Take $N \in \mathbb{N}^{*}$ and let $x_{0}^{k}:=r_{-}+\frac{(k-1)}{N}\left(r-r_{-}\right)$for $k=1 \cdots N$. 
- If $k=1$ then choose $\beta_{i}=\frac{(i-1)}{N}$ for $i=1 \cdots N$ and initialize (8.1) with (8.4) where $\beta$ is replaced by $\beta_{i}$.

- If $k>1$ initialize (8.1) with (8.2) where $y_{0}$ is replaced by $x_{0}^{k}$.

For both problems, the numerical integration of (8.1) is stopped when $t=T$ (with $T$ chosen sufficiently large). Any zero of the switching function $\phi$ (or equivalently $q$ ) during the numerical integration is marked by a dot point on the picture. These points correspond to switching points in the state space and to the switching curves (i.e. the loci where the control switches either from $u=0$ to $u=\bar{u}$ or from $u=\bar{u}$ to $u=0$ ).

\section{References}

[1] N.ApReutesei, An optimal control problem for a prey-predator system with a general functional response, Applied Mathematics Letters, Vol.22, N. 7, pp. 1062-1065, 2009.

[2] J.-P. Aubin, Viability Theory, Birkhäuser, 1991.

[3] J.-P. Aubin, A.M. Bayen, P. Saint-Pierre, Viability Theory, New Directions, Second Editions, Springer, 2011.

[4] T. Bayen, A. RApaport, About Moreau-Yosida regularization of the minimal time crisis problem, published online, Journal of Convex Analysis, vol. 23, 2016.

[5] T. Bayen, A. RAPAPORT, About the minimal time crisis problem, ESAIM, Proceedings and Surveys, to appear, 2017.

[6] J. Beddington And R. May, Maximum Sustainable Yields in Systems Subject to Harvesting at More than One Trophic Level Mathematical Biosciences, Vol. 51, 261-281, 1980.

[7] J. F. Bonnans, C. Moreno, Ch. Saguez, Controle de domaines temporels, INRIA research report 1984, https://hal.inria.fr/inria-00076249.

[8] J.F. Bonnans, V. Gaudrat, C. Saguez, A domain control approach to state constrained control problems, in Lecture Notes in Control and Information Sciences $\mathrm{n}^{o}$ 100, J.P. Zolesio ed., Springer-Verlag, Berlin,72-90, 1988.

[9] N. Bonneuil and K. Mullers, Viable Populations in a Prey-Predator System, Journal of Mathematical Biology, Vol. 35, pp. 261-293, 1997.

[10] L. Cesari, Optimization-Theory and Applications. Problems with Ordinary Differential Equations, Springer, 1983.

[11] F.H. Clarke, Functional Analysis, Calculus of Variation, Optimal control, Springer, 2013.

[12] F.H. Clarke, Y.S. Ledyaev, R.J. Stern, P.R. Wolenski, Nonsmooth Analysis and Control Theory, Springer-Verlag, New-York, 1998.

[13] M. De Lara And L. Doyen, Sustainable Management of Natural Resources. Mathematical Models and Methods, Springer 1998.

[14] M. De Lara, E. Ocana, R. Oliveros-Ramos And J. Tam, Ecosystem Viable Yields, Environmental Modeling \& Assessment, Vol. 17, N. 6, pp. 565-575, 2012.

[15] P. De Leenheer And D. Aeyels, Accessibility properties of controlled lotka-volterra systems, Proceedings of the 39th IEEE Conference on Decision and Control, Sydney, Australia, Vol. 4, 2000, pp. 3977-3981.

[16] L. Doyen, P. SAint-Pierre, Scale of viability and minimal time of crisis, Set-Valued Analysis 5, pp.227-246, 1997.

[17] M. Garavello, B. Piccoli, Hybrid Necessary Principle, SIAM J. Control Optim. Vol. 43, 5, pp. $1867-1887,2005$. 
[18] B.-S. GoH, Management and analysis of biological populations, Elsevier, 1980.

[19] B.-S. Goh, G. Leitmann And T. Vincent, Optimal control of a prey-predator system, Mathematical Biosciences, Vol. 19, N. 3-4, pp. 263-286, 1974.

[20] F. Grognard, Feedback stabilization of predator-prey systems for impulsive biological control, 19th IFAC World Congress, Cape Town, South Africa, pp. 5264-5269, 2014.

[21] F. Grognard, J. Rault and J.-L. Gouze, Positive control for global stabilization of predator-prey systems, 9th IFAC Symposium Nonlinear Control Systems (NOLCOS'13), Toulouse, France, pp.265-270, 2013.

[22] T. HABERKORN, E. TRÉLAT, Convergence results for smooth regularizations of hybrid nonlinear optimal control problems, SIAM . J. Control Optim., Vol. 49, 4, 1498-1522, 2011.

[23] A. Ibanez, Optimal control of the Lotka-Volterra system: turnpike property and numerical simulations, Journal of Biological Dynamics, Vol.11, N. 1, 2017.

[24] H. KhaliL, Nonlinear Systems, Macmillan Publishing Company, New York, 1992.

[25] L. Mailleret And F. Grognard, Global stability and optimisation of a general impulsive biological control model, Mathematical Biosciences, Vol. 221, N. 2, pp.91-100, 2009.

[26] M. Meza, A. Bhaya, E. Kaszkurewicz And M. Costa, Threshold policies control for predator-prey systems using a control Liapunov function approach, Theoretical Population Biology, Vol. 69, N. 4, pp. 273-284, 2005.

[27] L.S. Pontryagin, V.G. Boltyanskiy, R.V. Gamkrelidze, E.F. Mishchenko, Mathematical theory of optimal processes, The Macmillan Company, 1964.

[28] P. Prostyakov, Construction of the reachability set of the Lotka-Volterra system, Differ. Equ., Vol. 42, N. 3, pp. 391-399, 2006 (translation from Differ. Uravn., Vol 42, N. 3, pp. 366-373, 2006).

[29] M. Quincampoix, Differential inclusions and target problems, SIAM J. Control Optim., 30, 2, pp. 324$335,1992$.

[30] A. RAPAPORT, Characterization of barriers of differential games, Journal of Optimization, Theory and Applications, Vol. 97, No. 1, pp. 151-180, 1998.

[31] W. Strobele And H. WACKer, The economics of harvesting predator-prey systems, Journal of Economics, Vol. 61, N. 1, pp. 65-81, 1995.

[32] R. Vinter, Optimal Control, Systems and Control: Foundations and Applications, Birkhäuser, 2000.

[33] I. Yegorov, F. MAIREt, J.-L. Gouzé, Optimal feedback strategies for bacterial growth with degradation, recycling, and effect of temperature, Optim Control Appl Meth. 2018;39:1084?1109. https://doi.org/ 10.1002 /oca. 2398

[34] S. YosidA, An optimal control problem of the prey-predator system, Funck. Ekvacioj, Vol. 25, pp. 283-293, 1982.

[35] M. I. Zelikin, V. F. Borisov, Theory of Chattering Control, Systems \& Control: Foundations \& Applications, Birkhäuser, 1994.

[36] J. Zhu, E. Trélat, M. CERF, Planar tilting maneuver of a spacecraft: singular arcs in the minimum time problem and chattering, Discrete Cont. Dynam. Syst. Ser. B. 21 (2016), no. 4, pp. 1347-1388. 